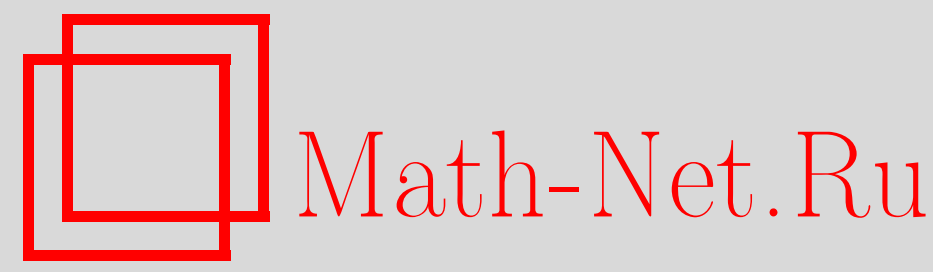

С. А. Назаров, А. С. Слуцкий, Асимптотика решений краевых задач для уравнения с быстроосциллирующими коэффициентами в области с малой полостью, Матем. сб., 1998, том 189, номер 9, 107-142

DOI: https://doi.org/10.4213/sm353

Использование Общероссийского математического портала Math-Net.Ru подразумевает, что вы прочитали и согласны с пользовательским соглашением

http://www . mathnet.ru/rus/agreement

Параметры загрузки:

IP: 54.80 .73 .141

26 апреля 2023 г., 10:44:10 


\author{
С.А. Назаров, А.С. Слуцкий
}

\title{
Асимптотика решений краевых задач для уравнения с быстроосциллирующими коэффициентами в области с малой полостью
}

\begin{abstract}
Найдены и обоснованы асимптотические представления решений краевых задач для уравнения второго порядка с быстроосциллирующими коэффиициентами в области с малой полостью (ее диаметр сравним с периодом осцилляции). На гранище полости назначаются условия Дирихле или Неймана. В дополнение к обычной для теории осреднения структуре асимптотического ряда возникают члены, описьвающие явление пограничного слоя вблизи отверстия, а решения осредненной задачи и их быстроосциллирующие корректоры приобретают особенности в точке, к которой стягиваются отверстия. Размерность области и другие факторы влияют даже на основной член асимптотики. Обсуждаются обобщения, в том числе и на систему уравнений теории упругости.
\end{abstract}

Библиография: 52 названия.

\section{§1. Постановка задач и предварительное описание результатов}

1. Краевые задачи. Пусть $n \geqslant 2, \Omega$ - параллелепипед в $\mathbb{R}^{n}$, определенный неравенствами $-a_{i}<x_{i}<a_{i}$, где $i=1,2, \ldots, n, x=\left(x_{1}, \ldots, x_{n}\right), a_{i}=p_{i} p_{0}^{-1}$, а $p_{0}, p_{1}, \ldots, p_{n}$ - натуральные числа. Пусть еще $\omega$ - область в $\mathbb{R}^{n}$ с компактным замыканием $\bar{\omega}$, содержашая начало координат $O$ и имеюшая гладкую границу. Обозначим через $\varepsilon=\left(p_{0} N\right)^{-1}$ малый положительный параметр $(N-$ большое натуральное число) и введем зависящие от $\varepsilon$ множества

$$
\omega_{\varepsilon}=\left\{x \in \mathbb{R}^{n}: y \equiv \varepsilon^{-1} x \in \omega\right\}, \quad \Omega(\varepsilon)=\Omega \backslash \bar{\omega}_{\varepsilon} .
$$

В области $\Omega(\varepsilon)$, содержащей малую полость $\omega_{\varepsilon}$, рассмотрим дифференциальное уравнение

$$
\mathscr{L}\left(x, \varepsilon^{-1} x, \partial_{x}\right) u(\varepsilon, x)=f\left(\varepsilon, x, \varepsilon^{-1} x\right), \quad x \in \Omega(\varepsilon) .
$$

Здесь $\partial_{x}=\operatorname{grad}$,

$$
\mathscr{L}\left(x, \varepsilon^{-1} x, \partial_{x}\right)=l_{0}\left(x, \frac{x}{\varepsilon}\right)-\sum_{i, j=1}^{n} \frac{\partial}{\partial x_{i}} l_{i j}\left(x, \frac{x}{\varepsilon}\right) \frac{\partial}{\partial x_{j}}
$$

функции $l . .(x, y)$ принадлежат классу $C^{\infty}\left(\bar{\Omega} \times[0,1]^{n}\right)$ и являются периодическими с периодами $2 a_{i}$ и 1 относительно $x_{i}$ и $y_{i}$ соответственно, $i=1, \ldots, n$ (далее такие функции называем гладкими, $x$ - и $y$-периодическими). Кроме того, $l_{0} \geqslant 0, l_{i j}=l_{j i}$ и

$$
\sum_{i, j=1}^{n} \xi_{i} l_{i j}(x, y) \xi_{j} \geqslant c_{0}|\xi|^{2} \quad \forall \xi \in \mathbb{R}^{n}, \quad x \in \bar{\Omega}, \quad y \in \mathbf{S} \equiv[0,1]^{n}
$$


причем $c_{0}$ - положительная постоянная. Наконец, правая часть $f$ уравнения (1.2) принадлежит $C^{\infty}\left(\left[0, \varepsilon_{0}\right) \times \bar{\Omega} \times \mathbf{S}\right)$ и является $y$-периодической; $\varepsilon_{0}>0$.

На $\partial \Omega$ поставим условия периодичности

где величины

$$
[u]_{i}=\left[\frac{\partial u}{\partial x_{i}}\right]_{i}=0, \quad x \in \Omega, \quad x_{i}=0 \quad(i=1, \ldots, n),
$$

равны скачку функции $v$ при переходе с грани $\Gamma_{i}^{+}$на грань $\Gamma_{i}^{-}$параллелепипеда $\Omega$, а $\Gamma_{i}^{ \pm}=\left\{x \in \bar{\Omega}: x_{i}= \pm a_{i}\right\}, e^{i}$ - орт оси $O x_{i}$.

На внутренней части $\partial \omega_{\varepsilon}$ границы $\partial \Omega(\varepsilon)$ назначим либо условия Дирихле

$$
u(\varepsilon, x)=0, \quad x \in \partial \omega_{\varepsilon},
$$

либо условия Неймана

$$
\mathscr{N}\left(x, \varepsilon^{-1} x, \partial_{x}\right) u(\varepsilon, x) \equiv \sum_{i, j=1}^{n} \nu_{i}\left(\frac{x}{\varepsilon}\right) l_{i j}\left(x, \frac{x}{\varepsilon}\right) \frac{\partial u}{\partial x_{j}}(\varepsilon, x)=0, \quad x \in \partial \omega_{\varepsilon} .
$$

В $(1.7) \nu=\left(\nu_{1}, \ldots, \nu_{n}\right)$ - единичный вектор внутренней нормали к $\partial \omega_{\varepsilon}$. Все данные задачи считаются гладкими, и условия (1.5)-(1.7) взяты однородными только для простоты.

Перечисленные задачи могут рассматриваться как модели сред с периодической структурой, имеюших малые изолированные дефекты. Нашей целью является построение асимптотики $u(\varepsilon, x)$ при $\varepsilon \rightarrow+0$. Асимптотическая структура решения сушественно зависит от размерности $n$ пространства и от свойств оператора (1.3). Поэтому описание результатов статьи, зависимое от вспомогательных утверждений, откладываем до п. 4.

2. Разрешимость задач. Предположим сначала, что

$$
l_{0}(x, y) \geqslant C_{0}>0 \quad \forall x \in \bar{\Omega}, \quad y \in \mathbf{S} .
$$

Обозначим через $\mathscr{H}^{1}(\Omega(\varepsilon))$ пространство $x$-периодических функций из класса Соболева $H^{1}(\Omega(\varepsilon))$. В силу $(1.4),(1.8)$ скалярным произведением в $H^{1}(\Omega(\varepsilon))$ может служить выражение

$$
\langle u, v\rangle_{\varepsilon}=\left(l_{0} u, v\right)_{\Omega(\varepsilon)}+\sum_{i, j=1}^{n}\left(l_{i j} \frac{\partial u}{\partial x_{j}}, \frac{\partial v}{\partial x_{i}}\right)_{\Omega(\varepsilon)},
$$

где $(\cdot, \cdot) \Xi-$ скалярное произведение в $L_{2}(\Xi)$. Величина $\langle u, u\rangle_{\varepsilon}^{1 / 2}$ является нормой в $H^{1}(\Omega(\varepsilon))$, эквивалентной

$$
\left(\left\|\partial_{x} u ; L_{2}(\Omega(\varepsilon))\right\|^{2}+\left\|u ; L_{2}(\Omega(\varepsilon))\right\|^{2}\right)^{1 / 2},
$$

причем константы эквивалентности не зависят от $\varepsilon \in\left(0, \varepsilon_{0}\right)$. Положим

$$
\mathscr{H}^{1}\left(\Omega(\varepsilon) ; \omega_{\varepsilon}\right)=\left\{v \in \mathscr{H}^{1}(\Omega(\varepsilon)): v=0 \text { на } \partial \omega_{\varepsilon}\right\} .
$$

Под обобшенным решением задачи $(1.2),(1.5),(1.7)$ подразумевается элемент $u$ пространства $\mathscr{H}^{1}(\Omega(\varepsilon))$, удовлетворяющий интегральному тождеству

$$
\langle u, v\rangle_{\varepsilon}=(f, v)_{\Omega(\varepsilon)} \quad \forall v \in \mathscr{H}^{1}(\Omega(\varepsilon)) .
$$

В случае условий Дирихле (1.6) и решение $u$, и пробные функции $v$ берутся из пространства $\mathscr{H}^{1}\left(\Omega(\varepsilon) ; \omega_{\varepsilon}\right)$. Следуюшее утверждение есть не что иное, как теорема Рисса о представлении непрерывного функционала в гильбертовом пространстве (см., например, [1]). 
ПРЕДЛОЖЕНИЕ 1.1. Если $f \in L_{2}(\Omega(\varepsilon))$ и выполнено условие (1.8), то существует единственное решение $u \in \mathscr{H}^{1}(\Omega(\varepsilon))$ задачи (1.2), (1.5); (1.6) или (1.7) и верна оченка

$$
\left\|u ; H^{1}(\Omega(\varepsilon))\right\| \leqslant c\left\|f ; L_{2}(\Omega(\varepsilon))\right\|
$$

c постоянной с, не зависящей от $f$ о от $\varepsilon \in\left(0, \varepsilon_{0}\right)$.

Пусть теперь

$$
l_{0}(x, y)=0 \quad \forall x \in \bar{\Omega}, \quad y \in \mathbf{S} ;
$$

тогда величина $\langle u, u\rangle_{\varepsilon}^{1 / 2}$ оказывается лишш полунормой в $\mathscr{H}^{1}(\Omega(\varepsilon))$. Она же является нормой в $\mathscr{H}^{1}\left(\Omega(\varepsilon) ; \partial \omega_{\varepsilon}\right)$ (благодаря условиям Дирихле $\left.(1.6)\right)$, но в неравенстве $\langle u, u\rangle_{\varepsilon}^{1 / 2} \geqslant c_{\varepsilon}\left\|u ; H^{1}(\Omega(\varepsilon))\right\|$ для $u \in \mathscr{H}^{1}\left(\Omega(\varepsilon) ; \partial \omega_{\varepsilon}\right)$ множитель $c_{\varepsilon}$ сушественно зависит от $\varepsilon$.

ПРЕДЛОЖЕНИЕ 1.2. Пусть $f \in L_{2}(\Omega(\varepsilon))$ и выполнено условие (1.13).

1) Решение $u \in \mathscr{H}^{1}(\Omega(\varepsilon))$ задачи (1.2), (1.5), (1.7) существует в том и только том случае, если $(f, 1)_{\Omega(\varepsilon)}=0$. Оно определено с точностью до постоянного слагаемого, а решение $u^{0}$, нормированное соотношением $\left(u^{0}, 1\right)_{\Omega^{0}}=0$, единственно и удовлетворяет оценке $(1.12)$ (при этом $\Omega^{0}$ фиксированная подобласть $\Omega$, не пересекающаяся с $\bar{\omega}_{\varepsilon}$ при $\varepsilon \in\left(0, \varepsilon_{0}\right)$, а левая часть (1.12) совпадает $c(1.10))$.

2) Существует единственное решение $u \in \mathscr{H}^{1}\left(\Omega(\varepsilon) ; \omega_{\varepsilon}\right)$ задачи (1.1), (1.5), (1.6); оно подчинено неравенству

$$
\left\|\partial_{x} u ; L_{2}(\Omega(\varepsilon))\right\|+\varepsilon^{-1+n / 2}\left\|R u ; L_{2}(\Omega(\varepsilon))\right\| \leqslant c \varepsilon^{1-n / 2}\left\|R^{-1} f ; L_{2}(\Omega(\varepsilon))\right\|,
$$

в котором $R(x)=r^{-n / 2}|\ln (r / a)|^{-1}, r=|x|, a=\left(a_{1}^{2}+\cdots+a_{n}^{2}\right)^{1 / 2}$ и постоянная с не зависит от $\varepsilon \in\left(0, \varepsilon_{0}\right)$ и от $f$.

ДокАЗАТЕЛЬство. В части существования и единственности решений можно сослаться на известные результаты (см. [1] и др.). Поэтому осталось проверить неравенство (1.14) или при учете (1.9), (1.13), (1.4) и (1.11) неравенство

$$
\left\|\partial_{x} u ; L_{2}(\Omega(\varepsilon))\right\|^{2} \geqslant c \varepsilon^{n-2}\left\|R u ; L_{2}(\Omega(\varepsilon))\right\|^{2},
$$

где $u \in \mathscr{H}^{1}\left(\Omega(\varepsilon) ; \partial \omega_{\varepsilon}\right)$. Продолжим функцию $u$ нулем на $\omega_{\varepsilon}$ и обозначим через $(r, \varphi)$ сферические координаты; точка $\varphi$ принадлежит единичной сфере $S^{n-1}$. Не ограничивая общности, считаем $u$ гладкой (в конце пользуемся замыканием по норме $\left.H^{1}\right)$ и пишем $u(r, \varphi)$. Имеем

$$
\begin{aligned}
& \int_{0}^{a(\varphi)} r^{-1}\left|\ln \frac{r}{a}\right|^{-2}|u(r, \varphi)|^{2} d r \\
& =2 \int_{0}^{a(\varphi)} r^{-1}\left|\ln \frac{r}{a}\right|^{-2} \int_{0}^{r} \frac{\partial u}{\partial \rho}(\rho, \varphi) u(\rho, \varphi) d \rho d r \\
& =2 \int_{0}^{a(\varphi)} \frac{\partial u}{\partial \rho}(\rho, \varphi) u(\rho, \varphi) \int_{\rho}^{a(\varphi)} r^{-1}\left|\ln \frac{r}{a}\right|^{-2} d r d \rho \\
& \leqslant 2 \int_{0}^{a(\varphi)}\left|\ln \frac{\rho}{a}\right|^{-1}\left|\frac{\partial u}{\partial \rho}(\rho, \varphi)\right||u(\rho, \varphi)| d \rho \\
& \leqslant 2\left(\int_{0}^{a(\varphi)}\left|\frac{\partial u}{\partial r}(r, \varphi)\right|^{2} r^{n-1} d r\right)^{1 / 2}\left(\int_{0}^{a(\varphi)} r^{-1}\left|\ln \frac{r}{a}\right|^{-2}|u(r, \varphi)|^{2} r^{2-n} d r\right)^{1 / 2}
\end{aligned}
$$


здесь $a(\varphi)$ - длина отрезка с началом $O$ и концом на $\partial \Omega$, лежащего на луче, который проходит через точку $\varphi$ на единичной сфере $S^{n-1}$. Так как $u(r, \varphi)=0$ при $r \leqslant c \varepsilon$ $\left(\right.$ внутри $\omega_{\varepsilon}$ ), то в последнем интеграле $r^{2-n} \leqslant c \varepsilon^{2-n}$ и, значит,

$$
\int_{0}^{a(\varphi)} r^{-n}\left|\ln \frac{r}{a}\right|^{-2}|u(r, \varphi)|^{2} r^{n-1} d r \leqslant c \varepsilon^{2-n} \int_{0}^{a(\varphi)}\left|\frac{\partial u}{\partial r}(r, \varphi)\right|^{2} r^{n-1} d r
$$

Проинтегрировав по $\varphi \in S^{n-1}$, приходим к (1.15).

Укажем оценки старших производных. Пусть $\mathscr{H}^{l}(\Omega(\varepsilon))$ - подпространство $x$-периодических функций из $H^{l}(\Omega(\varepsilon)), l=1,2, \ldots$, и $\partial_{x}^{\alpha}=\partial^{|\alpha|} / \partial x_{1}^{\alpha_{1}} \cdots \partial x_{n}^{\alpha_{n}},|\alpha|=$ $\alpha_{1}+\cdots+\alpha_{n}$.

ПрЕДЛОЖЕНИЕ 1.3. Пусть $u \in \mathscr{H}^{l+2}(\Omega(\varepsilon))$ - решение задачи (1.2), (1.5); (1.6) или (1.7) с правой частью $f \in \mathscr{H}^{l}(\Omega(\varepsilon))$. Тогда имеет место неравенcmso

$$
\begin{aligned}
& \sum_{1 \leqslant|\alpha| \leqslant l+2} \varepsilon^{2|\alpha|-2}\left\|\partial_{x}^{\alpha} u ; L_{2}(\Omega(\varepsilon))\right\|^{2} \\
& \leqslant c\left\{\sum_{|\alpha| \leqslant l} \varepsilon^{2|\alpha|+2}\left\|\partial_{x}^{\alpha} f ; L_{2}(\Omega(\varepsilon))\right\|^{2}+\left\|\partial_{x} u ; L_{2}(\Omega(\varepsilon))\right\|^{2}+\varepsilon^{2}\left\|u ; L_{2}(\Omega(\varepsilon))\right\|^{2}\right\}
\end{aligned}
$$

где постоянная с не зависит от $и$ и $\varepsilon \in\left(0, \varepsilon_{0}\right)$. В случае (1.13) последнее слагаемое из фигурных скобок мохнно удалить.

ДокАЗАТЕЛЬСтво. Возьмем какую-нибудь ячейку периодичности $\mathbf{S}_{\varepsilon}$ (сдвиг куба $[0, \varepsilon]^{n}$ на вектор $m^{\varepsilon}=\left(\varepsilon m_{1}, \ldots, \varepsilon m_{n}\right)$, где $m_{i}$ - целые числа) и обозначим через $\Sigma_{\varepsilon}$ объединение $\mathbf{S}_{\varepsilon}$ и всех других ячеек, примькающих к $\mathbf{S}_{\varepsilon}$, т.е. $\Sigma_{\varepsilon}=\{x$ : $\left.m_{i}-1 \leqslant \varepsilon^{-1} x_{i} \leqslant m_{i}+2\right\}$.

Пусть сначала $\Sigma_{\varepsilon}$ не пересекается с границей $\partial \Omega(\varepsilon)$. Положим

$$
\bar{u}(\varepsilon)=\left(\operatorname{mes}_{n} \Sigma_{\varepsilon}\right)^{-1} \int_{\Sigma_{\varepsilon}} u(\varepsilon, x) d x, \quad \widetilde{u}(\varepsilon, x)=u(\varepsilon, x)-\bar{u}(\varepsilon) .
$$

Функция $\widetilde{u}$ удовлетворяет уравнению

$$
\mathscr{L}\left(\varepsilon, x, \partial_{x}\right) \widetilde{u}(\varepsilon, x)=f(\varepsilon, x)-l_{0}\left(x, \varepsilon^{-1} x\right) \bar{u}(\varepsilon), \quad x \in \Sigma_{\varepsilon} .
$$

Перейдем к "быстрым" переменным $y=\varepsilon^{-1} x$ и применим локальные оценки решений эллиптических уравнений (см., например, [1], [2]). Вернувшись к прежним координатам $x$ и умножив полученное неравенство на $\varepsilon^{-2-n}$, находим

$$
\begin{aligned}
& \sum_{|\alpha| \leqslant l+2} \varepsilon^{2|\alpha|-2}\left\|\partial_{x}^{\alpha} \widetilde{u} ; L_{2}\left(\mathbf{S}_{\varepsilon}\right)\right\|^{2} \\
& \leqslant c\left\{\varepsilon^{-2}\left\|\widetilde{u} ; L_{2}\left(\Sigma_{\varepsilon}\right)\right\|^{2}+\varepsilon^{2}\left(\sum_{|\alpha| \leqslant l} \varepsilon^{2|\alpha|}\left\|\partial_{x}^{\alpha} f ; L_{2}\left(\Sigma_{\varepsilon}\right)\right\|^{2}+\left\|\bar{u} ; L_{2}\left(\Sigma_{\varepsilon}\right)\right\|^{2}\right)\right\} .
\end{aligned}
$$


Поскольку $\widetilde{u}$ обладает нулевым средним по $\Sigma_{\varepsilon}$, то справедливо неравенство Пуанкаре

$$
\int_{\Sigma_{\varepsilon}}\left|\partial_{x} \widetilde{u}(\varepsilon, x)\right|^{2} d x \geqslant c \varepsilon^{-2} \int_{\Sigma_{\varepsilon}}|\widetilde{u}(\varepsilon, x)|^{2} d x
$$

(см. [1]) и, следовательно, в силу очевидных соотношений $\partial_{x}^{\alpha} \widetilde{u}=\partial_{x}^{\alpha} u,|\alpha| \geqslant 1$, и $\left\|\bar{u} ; L_{2}\left(\Sigma_{\varepsilon}\right)\right\|^{2} \leqslant c\left\|u ; L_{2}\left(\Sigma_{\varepsilon}\right)\right\|^{2}$ неравенство (1.19) переписьваем в виде

$$
\begin{aligned}
& \sum_{1 \leqslant|\alpha| \leqslant l+2} \varepsilon^{2|\alpha|-2}\left\|\partial_{x}^{\alpha} u ; L_{2}\left(\mathbf{S}_{\varepsilon}\right)\right\|^{2} \\
& \leqslant c\left\{\sum_{|\alpha| \leqslant l} \varepsilon^{2|\alpha|+2}\left\|\partial_{x}^{\alpha} f ; L_{2}\left(\Sigma_{\varepsilon}\right)\right\|^{2}+\left\|\partial_{x} u ; L_{2}\left(\Sigma_{\varepsilon}\right)\right\|^{2}+\left\|u ; L_{2}\left(\Sigma_{\varepsilon}\right)\right\|^{2}\right\}
\end{aligned}
$$

Перейдем к рассмотрению запрешенных случаев. Если $\partial \Omega \cap \Sigma_{\varepsilon} \neq \varnothing$, то ввиду $x$-периодичности $u$ и $f$ нужная перезапись оценки (1.21) затруднений не вызывает. Пусть $\partial \omega_{\varepsilon} \cap \Sigma_{\varepsilon} \neq \varnothing$ (подчеркнем, что количество таких ячеек $\mathbf{S}_{\varepsilon}$ не зависит от $\varepsilon$; см. (1.1)). При задании на $\partial \omega_{\varepsilon}$ условий Неймана (1.7) все выкладки (1.17)-(1.21) сохраняются, но в них приходится $\Sigma_{\varepsilon}$ и $\mathbf{S}_{\varepsilon}$ заменить на $\Sigma_{\varepsilon} \backslash \omega_{\varepsilon}$ и $\mathbf{S}_{\varepsilon} \backslash \omega_{\varepsilon}$, а при употреблении локальных оценок - принять во внимание равенство $\mathscr{N}\left(\varepsilon, x, \partial_{x}\right) \widetilde{u}(\varepsilon, x)=0, x \in \partial \omega_{\varepsilon} \cap \Sigma_{\varepsilon}$. В случае условий Дирихле (1.6) достаточно положить $\widetilde{u}=u$ и $u=0$, произвести те же самые замены множеств и вывести (1.20) из неравенства Фридрихса (так как $u=0$ на $\partial \omega_{\varepsilon} \cap \Sigma_{\varepsilon} \neq \varnothing$ ).

Итак, после упомянутых модификаций неравенство (1.21) оказывается верным для всех ячеек, причем постоянную $c$ можно выбрать общей. Теперь формула (1.16) получается суммированием (1.21) при учете конечной кратности покрытия $\Omega$ множествами типа $\Sigma_{\varepsilon}$.

При $l_{0}=0$ вычитаемое справа в (1.18) исчезает, а в (1.19) и (1.21) нет $\left\|\bar{u} ; L_{2}\left(\Sigma_{\varepsilon}\right)\right\|$ и $\left\|u ; L_{2}\left(\Sigma_{\varepsilon}\right)\right\|$ - это подтверждает последнюю фразу в формулировке предложения 1.3 .

3. Предельные задачи. Одной из базовых идей теории осреднения (см. [3]-[8] и др.) является разделение зависимостей от "медленных" $x$ и “быстрых" $y=\varepsilon^{-1} x$ переменных. В частности, после такого разделения естественным образом возникает уравнение на ячейке

$$
L_{0}\left(x, y, \partial_{y}\right) v(x, y)=h(x, y), \quad y \in \mathbf{S}=[0,1]^{n}
$$

параметрически зависяшее от $x \in \Omega$ и рассматриваемое в классе $y$-периодических функций, где

$$
L_{0}\left(x, y, \partial_{y}\right)=-\sum_{i, j=1}^{n} \frac{\partial}{\partial y_{i}} l_{i j}(x, y) \frac{\partial}{\partial y_{j}}
$$

В следующем утверждении (см. [9] и ср. с предложениями $1.2,1)$ и 1.3$)$ фигурирует пространство $\mathscr{H}^{l}(\mathbf{S})$, составленное из $y$-периодических функций, а зависимость от $x \in \bar{\Omega}$ считается (и оказывается) гладкой. 
ПРЕДЛОЖЕНИЕ 1.4. Пусть $h \in \mathscr{H}^{l}(\mathbf{S}), l=0,1, \ldots$ Решение $v \in \mathscr{H}^{l+2}(\mathbf{S})$ уравнения (1.22) существует в том и только том случае, если

$$
\langle h\rangle \equiv(h, 1)_{\mathbf{S}}=0 .
$$

Решение определено с точностью до постоянного слагаемого; при выполнении условия нормировки $\langle v\rangle=0$ оно становится единственным.

Далее под решением уравнения (1.22) всегда подразумевается $y$-периодическая функция с нулевым средним по $\mathbf{S}$.

В результате применения обычной процедуры осреднения (см. [3]-[8] и др.) появляется дифференциальньй оператор

$$
\mathbf{L}\left(x, \partial_{x}\right)=\mathbf{l}_{0}(x)-\sum_{i, j=1}^{n} \frac{\partial}{\partial x_{i}} \mathbf{l}_{i j}(x) \frac{\partial}{\partial x_{j}},
$$

освобожденный от быстрых переменных и имеющий такие коэффициенты:

$$
\mathbf{l}_{0}=\left\langle l_{0}\right\rangle, \quad \mathbf{l}_{i j}=\left\langle l_{i j}\right\rangle+\sum_{p=1}^{n}\left\langle l_{i p} \frac{\partial Y_{j}}{\partial y_{p}}\right\rangle ;
$$

здесь $Y_{j}(x, y)$ - решение уравнения (1.22) с правой частью

$$
h_{j}(x, y)=\sum_{i=1}^{n} \frac{\partial l_{i j}}{\partial y_{i}}(x, y) .
$$

Известно (см. [3]-[8]), что для коэффициентов (1.26) справедливы условие типа (1.4) и равенство $\mathbf{l}_{i j}(x)=\mathbf{l}_{j i}(x)$. Поэтому верны формулируемые ниже утверждения о предельной задаче:

$$
\begin{gathered}
\mathbf{L}\left(x, \partial_{x}\right) U(x)=F(x), \quad x \in \Omega ; \\
{[U]_{i}=\left[\frac{\partial U}{\partial x_{i}}\right]_{i}=0, \quad x \in \Omega, \quad i=1, \ldots, n .}
\end{gathered}
$$

ПРЕДЛОЖЕНИЕ 1.5. 1) В случае (1.8) существует единственное решение $U \in \mathscr{H}^{1}(\Omega)$ задачи (1.28) с правой частью $F \in L_{2}(\Omega)$.

2) При выполнении (1.13) решение $U \in \mathscr{H}^{1}(\Omega)$ задачи (1.28) с $F \in L_{2}(\Omega)$ существует тогда и только тогда, когда $(F, 1)_{\Omega}=0$. Оно определено $c$ точностью до постоянного слагаемого.

3) Если $F \in \mathscr{H}^{l}(\Omega)$ при некотором $l=0,1, \ldots$, mо $U \in \mathscr{H}^{l+2}(\Omega)$.

Наконец, явление пограничного слоя вблизи отверстия $\omega_{\varepsilon}$ описьвается при помощи решений уравнения

$$
L_{0}\left(0, y, \partial_{y}\right) w(y)=\mathbf{F}(y), \quad y \in \mathbb{R}^{n} \backslash \bar{\omega},
$$

снабженного краевыми условиями

$$
w(y)=\mathbf{G}(y), \quad y \in \partial \omega,
$$

или

$$
N_{0}\left(0, y, \partial_{y}\right) w(y) \equiv \sum_{i, j=1}^{n} \nu_{i}(y) l_{i j}(0, y) \frac{\partial w}{\partial y_{j}}(y)=\mathbf{G}(y), \quad y \in \partial \omega,
$$

соответствуюшими (1.6) или (1.7). Вопрос о разрешимости задач $(1.29),(1.30)$ и (1.29), (1.31) обсуждается в п. $3 \S 2$. 
4. Описание результатов. Планомерное изучение эллиптических задач в областях с сингулярными возмущениями в виде малых полостей и отверстий начато работами А. М. Ильина [10]-[12] (см. также [13]) - в них применялся метод сращиваемых разложений и были построены и обоснованы асимптотические представления решений различных краевых задач для уравнений второго порядка. Иной, хотя и близкий по духу, метод, центральным моментом в котором является процедура перераспределения невязок (см. ниже), был предложен В. Г. Мазья, С. А. Назаровым, Б. А. Пламеневским [14], [15] и затем применен для упомянутого класса сингулярно возмущенных задач в [16]-[21] (см. также [22], [23]), где помимо асимптотик решений общих краевых задач для эллиптических систем изучались асимптотики собственных чисел и вектор-функций, энергетических функционалов и пр.

Основным техническим элементом обоих методов служит обработка ("сращивание" или “перераспределение") разложений функций около точки $O$ и на бесконечности. При отсутствии осцилляции подобные разложения решений вытекают из общих результатов В. А. Кондратьева [24] (см. также [25]-[29] и др.) об эллиптических задачах в областях с коническими точками. В нашем случае та же теория позволяет получить разложения вблизи точки $O$ решений осредненной задачи (1.28). В ячеечном уравнении (1.22) медленные переменные $x$ выступают как параметр, и нужные разложения по $r=|x|$ доступны даже без ссылок на [24]. Однако в уравнении (1.29) коэффициенты оператора $L_{0}\left(0, y, \partial_{y}\right)$ не стабилизируются на бесконечности, теория В. А. Кондратьева оказывается неприменимой, а вопрос о поведении при $|y| \rightarrow \infty$ решений $w(y)$ типа пограничного слоя решается отдельно, и ответ на него извлекается из статей С. А. Назарова [30], [31].

Все используемые далее разложения решений $U, v$ и $w$ предельных задач сообщаются в $\S 2$. В остальных параграффах для задачи (1.2), (1.5); (1.6) или (1.7) выводятся результаты того же свойства, что и в [11], [12], [22; гл. 1], [23; гл. 2] для случая гладких коэффициентов. Вкупе с упоминавшейся процедурой перераспределения невязок применяется метод построения полных асимптотических рядов для решений задач с быстроосциллирующими коэффициентами, предложенный Н. С. Бахваловым в [32] (см. также [3]-[8] и [33], [34]). Тем не менее, простое совмешение двух подходов не приводит к успеху - требуются новые соображения, что вызвано следующими двумя обстоятельствами. Во-первых, при реализации итерационных процессов из [32] происходит “потеря гладкости”, т.е. определение асимптотического члена $U_{k}$ гладкого типа зависимо от старших производных предыдущих членов $U_{k-1}, \ldots, U_{0}$, которые (члены), как станет ясно далее, в нашей ситуации могут даже терять непрерывность в точке $O$. Во-вторых, разложения на бесконечности решений $w_{k}(y)$ типа пограничного слоя отягощены двойной, степенной и периодической, зависимостью от $y$, и употребление процедуры перераспределения невязок сталкивается с трудностями, не встречавшимися в [14], [15], [22], [23] (ср. с [35]-[37]). Все это требует модификации названных алгорифмов, хотя по виду асимптотический ряд

$$
\begin{aligned}
u(x, \varepsilon)=\sum_{k=\mu}^{\infty} \varepsilon^{k}\left\{U_{k}(x, \log \varepsilon)\right. & +\varepsilon \sum_{j=1}^{3} Y_{j}\left(x, \frac{x}{\varepsilon}\right) \frac{\partial U_{k}}{\partial x_{j}}(x, \log \varepsilon) \\
& \left.+\varepsilon^{2} v_{k}\left(x, \frac{x}{\varepsilon}, \log \varepsilon\right)+\chi(x) w_{k}\left(\frac{x}{\varepsilon}, \log \varepsilon\right)\right\}
\end{aligned}
$$


не отличается от привычного: в $(1.32) U_{k}$ - решения осредненной задачи в области без отверстия, два следующих члена представляют собой быстроосциллирующие корректоры, а последний является решением типа пограничного слоя около $\partial \omega_{\varepsilon}$. Подчеркнем, что только основные члены $U_{0}$ и $w_{0}$ определяются по прежним схемам из [32] и [22], [23].

Обрисуем структуру статьи. В следующем, втором, параграфе собраны известные факты о поведении решений предельных задач вблизи проколотой точки $x=0$ или на бесконечности; попутно вводятся специальные решения внешних задач $(1.29),(1.30)$ и $(1.29),(1.31)$ и определяются интегральные характеристики множества $\omega$, связанные с оператором $L_{0}\left(0, y, \partial_{y}\right)$.

В $\S 3$ мы приступаем собственно к асимптотическому анализу - изучается задача Дирихле в трехмерной области. Сначала при условии $l_{0}>0$ (все предельные задачи однозначно разрешимы) строится и обосновывается главный член асимптотики решения, а затем определяются все коэффициенты ряда (1.32) и производится сравнение его частичной суммы с истинным решением задачи $(1.2),(1.5),(1.6)$ по энергетической метрике (оценки старших производных обсуждаются в п. $1 \S 6$ ). Наконец, в п. $4 \S 3$ рассматривается оператор (1.3) с нулевым свободным членом $l_{0}$; в отличие от предыдущего случая, для которого $\mu=0$ в (1.32), здесь из-за неоднозначной разрешимости задачи (1.28) появляется "растушее" слагаемое $\varepsilon^{-1} c_{0}$, т.е. оказывается, что $\mu=-1$ в (1.32) (для оператора второго порядка с гладкими коэффициентами подобный эффект отражен в [12], [13], а для общих эллиптических задач - в [15], [21], [23]). Подчеркнем еше, что случай $n>3$ лишь незначительными деталями отличается от $n=3$.

Четвертый параграф посвяшен двумерной задаче Дирихле, для которой при $l_{0} \neq 0$ асимптотические конструкции усложняются ввиду рациональной зависимости всех, в том числе и основных, членов ряда (1.32) от параметра $\log \varepsilon$ (впервые для уравнения второго порядка с гладкими коэффициентами такая зависимость была обнаружена в [11]; обобшение этого результата на системы можно найти в [15], [21], [23]). Если же $l_{0}=0$, то, по-прежнему, слагаемые из $(1.32)$ - полиномы от $\log \varepsilon ;$ кроме того, в обеих ситуациях $\mu=0$. Подобное варьирование структуры асимптотического ряда, обусловленное изменением $n$ или $l_{0}$, наблюдается и для задачи Неймана $(1.2),(1.5),(1.7)$, обсуждаемой в $\S 5$. Кроме того, в $\S 5$ приводятся различные обобшения постановок задач и реализации итерационных процессов: краевые условия Дирихле на границе $\partial \Omega$ или ее части, эллиптические системы уравнений, удаление нескольких малых отверстий и т. п.

\section{§2. Асимптотические разложения решений предельных задач}

1. Ячеечное уравнение. Для укорачивания формул примем соглашение: запись $a(x, y)=a_{0}(x, y)+o\left(r^{\Lambda-0}\right)$ означает, что для любых мультииндексов $\alpha, \gamma$ и положительного $\delta$ справедливо соотношение

$$
\left|\partial_{x}^{\alpha} \partial_{y}^{\gamma} a(x, y)-\partial_{x}^{\alpha} \partial_{y}^{\gamma} a_{0}(x, y)\right| \leqslant c_{\alpha \gamma \delta} r^{\Lambda-|\alpha|-\delta} \quad \forall x \in \bar{\Omega}, \quad y \in \mathbf{S}
$$

Коэффиициенты $l_{i j}$ из (1.23) гладко зависят от параметра $x$. Поэтому оператор $L_{0}$ раскладывается в ряд Тейлора в точке $x=0$, и следующее утверждение становится очевидньм. 
ПРЕДЛОЖЕНИЕ 2.1. Если для правой части $h$ верна формула

$$
h(x, y)=\sum_{j=\mu}^{J} r^{j} h^{(j)}(\varphi, \log r, y)+o\left(r^{J+1-0}\right)
$$

то аналогичное представление

$$
v(x, y)=\sum_{j=\mu}^{J} r^{j} v^{(j)}(\varphi, \log r, y)+o\left(r^{J+1-0}\right)
$$

выполняется и для решения $v$ уравнения (1.22). Здесь $\mu-$ иелое число, $J=$ $\mu, \mu+1, \ldots ;(r, \varphi)$ - сферические координатьи, $r=|x|, \varphi \in S^{n-1} ;$ функиии $h^{(j)}$ u $v^{(j)}$ зависят гладко от $\varphi \in S^{n-1}$, полиномиально от $\log r$ и гладко периодически от $y \in \mathbf{S}$, причем их средние по $\mathbf{S}=[0,1]^{n}$ равны нулю.

Всюду далее имеем дело со случаем $\mu=-2$.

2. Осредненная задача. Принимая проколотоепространство $\mathbb{R}^{n} \backslash O$ за конус, конкретизируем результаты теории эллиптических задач в областях с коническими точками.

Tеорема 2.2 (см. [24], [25], а также [27]-[29]). Пусть

$$
F(x)=\sum_{j=0}^{J} r^{j-2} F^{(j)}(\varphi, \log r)+o\left(r^{J-1-0}\right)
$$

тогда решение $U$ задачи (1.28), подчиненное условию $U(x)=o\left(r^{-0}\right)$, обладает такой асимптотикой при $r \rightarrow 0$ :

$$
U(x)=\sum_{j=0}^{J} r^{j} U^{(j)}(\varphi, \log r)+o\left(r^{J+1-0}\right) .
$$

Здесь всюду обозначения аналогичны использованным в предлоэсении 2.1.

Функции $F$ и $U$, упомянутые в теореме 2.2 , принадлежат $L_{2}(\Omega)$ и $H^{1}(\Omega)$ только в случаях $n>4$ и $n>2$ соответственно. Это вступает в противоречие с формулировкой предложения 1.5. При $n \geqslant 3$ ситуация исправляется при помощи известной оценки

$$
\left\|u ; H^{1}(\Omega)\right\| \geqslant c\left\|r^{-1} u ; L_{2}(\Omega)\right\|,
$$

вытекающей из неравенства Харди (см., к примеру, §2.4 в [28] или [29]) и обеспечиваюшей сушествование решения $U \in H^{1}(\Omega)$ задачи (1.28) при ограничении $r F \in L_{2}(\Omega)$, которому функция из (2.3) уже удовлетворяет. При $n=2$ вместо $(2.5)$ имеем такую оценку:

$$
\left\|u ; H^{1}(\Omega)\right\| \geqslant c\left\|r^{-1}\left|\ln \frac{r}{a}\right|^{-1} u ; L_{2}(\Omega)\right\|
$$


Она следует из известного одномерного неравенства Харди

$$
\int_{0}^{a(\varphi)} r^{-1}\left|\ln \frac{r}{a}\right|^{-2}|u(r, \varphi)|^{2} d r \leqslant 4 \int_{0}^{a(\varphi)} r\left|\frac{\partial u}{\partial r}(r, \varphi)\right|^{2} d r
$$

которое было установлено, по существу, при проверке (1.15). Значит, утверждения $1.5,1), 2)$ остаются в силе при условии $r|\ln (r / a)| F \in L_{2}(\Omega)$, однако такое расширение сферы действия результата недостаточно, так как для $F$ из (2.3) указанное включение может нарушаться. Здесь нас спасает стандартный прием, описанный, например, в гл. 2 [28] или [29]: сначала при помощи леммы 3.5.11 из этих книг строится частное решение $U^{(0)}(\varphi, \log r)$ уравнения

$$
-\sum_{i, j=1}^{n} \frac{\partial}{\partial x_{i}} \mathbf{l}_{i j}(0) \frac{\partial}{\partial x_{j}} U^{(0)}=r^{-2} F^{(0)} \text { в } \mathbb{R}^{2} \backslash O,
$$

затем оно умножается на срезку $\chi \in C_{0}^{\infty}(\Omega)$, равную 1 вблизи точки $O$, и, наконец, при использовании предложения 1.5 отыскивается решение $\widetilde{U} \in \mathscr{H}^{1}(\Omega)$ задачи $(1.28)$ с новой правой частью $F-\mathbf{L} \chi U^{(0)}$, подчиненной нужному условию, сумма $U=\widetilde{U}+\chi U^{(0)}$ как раз удовлетворяет (1.28). Нетрудно убедиться в том, что решение однородного уравнения $(2.7)$ с нужным поведением при $r \rightarrow 0$ имеет вид

$$
c_{0}+c_{1}\{-\sigma \log r+\psi(\varphi)\},
$$

где $c_{i}$ - произвольные постоянные, а в фигурных скобках располагается фундаментальное решение для оператора (2.7). Подчеркнем, что за счет выбора множителя $c_{1}$ в аддитивной составляющей (2.8) функции $U^{(0)}$ можно найти решение $U$ даже при нарушении равенства $(F, 1)_{\Omega}=0$ (cp. с предложением $\left.1.5,2\right)$ и см. подробности в $\S 2.4$ [28] или [29]). Итак, указанная процедура приводит к утверждению, обобшаюшему предложение 1.5 .

СлЕДСТВИЕ 2.3. Пусть $n=2$ и верна формула (2.3). Тогда существует решение $U$ задачи (1.28), представимое в виде (2.4). В случае (1.13) оно определено с точностью до постоянного слагаемого, а в случае (1.8) - с точностью до слагаемого const $G(x)$, где $G$ - функиия Грина задачи (1.28) с особенностью в точке $O$.

3. Задачи о пограничном слое. Вопрос о разрешимости задач (1.29), (1.30) и (1.29), (1.31) изучен в полной мере (см., например, [38]), и мы ограничиваемся формулировками соответствуюших утверждений.

Обозначим через $\mathscr{D}$ пополнение $C_{0}^{\infty}\left(\mathbb{R}^{n} \backslash \omega\right)$ по норме интеграла Дирихле $\left\|\partial_{x} u ; L_{2}\left(\mathbb{R}^{n} \backslash \omega\right)\right\|$. Из уже упоминавшихся одномерных неравенств Харди вытекают соотношения

$$
\begin{gathered}
\left\|\partial_{y} u ; L_{2}\left(\mathbb{R}^{n} \backslash \omega\right)\right\| \geqslant c\left\|\rho^{-1} u ; L_{2}\left(\mathbb{R}^{n} \backslash \omega\right)\right\|, \quad n \geqslant 3 ; \\
\left\|\partial_{y} u ; L_{2}\left(\mathbb{R}^{2} \backslash \omega\right)\right\|+\left\|u ; L_{2}\left(B_{R_{e}} \backslash \omega\right)\right\| \geqslant c\left\|\rho^{-1}\left|\ln \frac{\rho}{R_{i}}\right|^{-1} ; L_{2}\left(\mathbb{R}^{2} \backslash \omega\right)\right\| ;
\end{gathered}
$$

здесь $u \in \mathscr{D}, \rho=|y|$ и $B_{R}=\left\{y \in \mathbb{R}^{n}:|y|<R\right\}$, причем $\bar{\omega} \subset B_{R_{e}}$ и $B_{R_{i}} \subset \omega$. Поскольку, используя подходящее продолжение функции $\mathbf{G}$, краевые условия можно 
сделать однородными, будем считать, что $\mathbf{G}=0$ в (1.30) и (1.31). Под обобщенным решением задачи $(1.29),(1.31)$ понимается элемент $w$ пространства $\mathscr{D}$, удовлетворяющий интегральному тождеству

$$
\sum_{i, j=1}^{n}\left(l_{i j}(0, \cdot) \frac{\partial w}{\partial x_{j}}, \frac{\partial v}{\partial x_{i}}\right)_{\mathbb{R}^{n} \backslash \omega}=(\mathbf{F}, v)_{\mathbb{R}^{n} \backslash \omega} \quad \forall v \in \mathscr{D} .
$$

Для задачи Дирихле дополнительно требуется, чтобы $w$ и $v$ аннулировались на $\partial \omega$.

ПРЕДЛОЖЕНИЕ 2.4. Если $n>2$ u $\rho \mathbf{F} \in L_{2}\left(\mathbb{R}^{n} \backslash \omega\right), \mathbf{G}=0$, то существует единственное решение $w \in \mathscr{D}$ задачи (1.29), (1.30) (или задачи (1.29), (1.31)).

ПРЕДЛОЖЕНИЕ 2.5. Пусть $n=2 u \rho\left|\ln \left(\rho / R_{i}\right)\right| \mathbf{F} \in L_{2}\left(\mathbb{R}^{2} \backslash \omega\right), \mathbf{G}=0$. При этих условиях

1) существует единственное решение $w \in \mathscr{D}$ задачи (1.29), (1.30);

2) задача (1.29), (1.31) имеет решение $w \in \mathscr{D}$ тогда и только тогда, когда $(\mathbf{F}, 1)_{\mathbb{R}^{2} \backslash \omega}=0 ;$ оно определено с точностью до постоянного слагаемого.

В [30], [31] построена и обоснована асимптотика на бесконечности решения задачи Дирихле для системы уравнений второго порядка в плоской области с угловым выходом на бесконечность. Отсутствие у границы $\partial \omega$ некомпактной компоненты упрощает как саму процедуру отыскания асимптотики (нет пограничных слоев около сторон угла), так и рассуждения, приводящие к оправданию разложений. Ограничение $n=2$ имело чисто геометрическую природу и связывалось опять-таки с неудобными краевыми эффектами. Перечисленные обстоятельства позволяют без труда перенести результаты [30], [31] не только на уравнение (1.29) при $n=2$, но и на многомерные внешние задачи Дирихле и Неймана.

Процедура [30], [31] отыскания разложений при $\rho=|y| \rightarrow \infty$ во многом напоминает обычный алгорифм построения асимптотического ряда для решения дифференциального уравнения с быстроосциллирующими коэффициентами (к примеру, сферические координаты $(\rho, \varphi)$ объявляются медленными, а декартовы $y$ - быстрыми). В следующих параграфах понадобится только сам результат - разложение решения, и потому сопутствующие конструкции мы здесь не затрагиваем.

ПРЕДЛОЖЕНИЕ 2.6. Пусть $\mathbf{G} \in C^{\infty}(\partial \omega)$, а для правой части $\mathbf{F}$ уравнения (1.29) при любьх мультииндексе $\alpha$ и положстельном $\delta$ верна формула

$$
\left|\partial_{y}^{\alpha}\left\{\mathbf{F}(y)-\sum_{j=1}^{J} \rho^{-j-2}\left[\mathbf{F}^{(j)}(\varphi, \log \rho)+\mathbf{H}^{(j)}(\varphi, \log \rho, y)\right]\right\}\right| \leqslant c_{\alpha \delta} \rho^{-J-3+\delta}
$$

Тогда для исчезающего на бесконечности решения $ш$ задачи (1.29), (1.30) или (1.29), (1.31) справедливо соотношение

$$
\left|\partial_{y}^{\alpha}\left\{w(y)-\sum_{j=1}^{J} \rho^{-j}\left[w^{(j)}(\varphi, \log \rho)+W^{(j)}(\varphi, \log \rho, y)\right]\right\}\right| \leqslant c_{\alpha \delta} \rho^{-J-1+\delta}
$$


причем $W^{(j)}(\cdot, \cdot, y)$ имеют нулевъе средние по $y \in \mathbf{S}$ и допускают представление

$$
\begin{aligned}
\rho^{-j} W^{(j)}(\varphi, \log \rho, y)= & \rho^{-j} \mathbf{W}^{(j)}(\varphi, \log \rho, y) \\
& \quad+\sum_{i=1}^{n} Y_{i}(0, y) \frac{\partial}{\partial y_{i}}\left[\rho^{-j+1} w^{(j-1)}(\varphi, \log \rho)\right]
\end{aligned}
$$

Наконеи, $\mathbf{W}^{(1)}=\mathbf{W}^{(2)}=0$, а обозначения в (2.10)-(2.12) аналогичнь использованным в предложсениях 2.1, 2.2 и в $n .3 \S 1$.

ЗАмЕчАниЕ 2.7. Если $\mathbf{F}$ и $w$ заменить рядами из (2.10) и (2.11) (положить там $J=\infty)$ и приравнять члены порядка $O\left(\rho^{-j-2}\right)$ слева и справа в $(1.29)$, то после выгисления среднего по третьему аргументу $y \in \mathbf{S}$ получится уравнение вида $(2.7)$ для $\rho^{-j} w^{(j)}$, а оставшаяся (с нулевым средним) часть равенства дает уравнение на $\mathbf{S}$ с оператором $L_{0}\left(0, y, \partial_{y}\right)$ для определения $\rho^{-j-2} \mathbf{W}^{(j+2)}$ (увеличение индекса на 2 согласуется с соотношениями $\mathbf{W}^{(1)}=\mathbf{W}^{(2)}=0$ ). Такая же картина наблюдается и в классической процедуре осреднения.

ЗАмечАнИЕ 2.8. Из-за того, что у членов сумм по $j$ в $(2.10)$ и (2.11) множителями служат $y$-периодические функции $\mathbf{H}^{(j)}$ и $W^{(j)}$, оценки остатков, указанные в предложении 2.6, имеют иное качество, нежели в предложениях 2.1 и 2.2: они (остатки) убьвают на бесконечности с той же скоростью, что и любые их производные, - именно так ведет себя первый (с номером $J+1)$ из отброшенных членов.

Асимптотические формулы для решений, обслуживающие потребности асимптотического анализа исходной задачи, не всегда согласуются с утверждениями о разрешимости предельных задач в энергетических классах. Так, функция $\mathbf{F}$, фигурируюшая в (2.10), удовлетворяет условиям предложений 2.4, 2.5 только при $n \leqslant 3$. Впрочем, для $n>3$ положение дел исправляется при помощи того же приема, что и в п. $2,-$ построения частного решения, компенсируюшего слагаемые $\rho^{-j-2}\left(\mathbf{F}^{(j)}+\mathbf{H}^{(j)}\right)$ c $j \leqslant(n-2) / 2$ в представлении для $\mathbf{F}$.

Другая неувязка состоит в том, что предложение 2.5 , относящееся к случаю $n=2$, вовсе не гарантирует сушествование убывающих при $|y| \rightarrow \infty$ решений задач $(1.29),(1.30)$ и (1.29), (1.31). С помощью таких решений описывается явление пограничного слоя около $\omega_{\varepsilon}$, для которого малость на удалении от $\omega_{\varepsilon}-$ неотъемлемое свойство. Иными словами, совершенно необходимо определить условия сушествования убываюшего решения $w$. Задачей Дирихле мы займемся в следуюшем разделе, а сейчас обратимся к задаче Неймана (1.29), (1.31).

Опираясь на результаты [30], [31], нетрудно заключить, что при $n=2$ для функции $\omega \in \mathscr{D}$, удовлетворяющей уравнению (1.29) с правой частью $\mathbf{F}$ из $(2.10)$, справедливы неравенства (2.11), где $|\alpha|>0$, но при $\alpha=(0,0)$ неравенство (2.11) заменяется таким:

$$
\left|w(y)-c_{\omega}-\sum_{j=1}^{J} \rho^{-j}\left[w^{(j)}(\varphi, \log \rho)+W^{(j)}(\varphi, \log \rho, y)\right]\right| \leqslant c_{0 \delta} \rho^{-J-1-\delta}
$$

здесь $c_{\omega}$ - постоянная, зависящая от $\omega$. Так как $1 \in \mathscr{D}$ - решение однородной задачи (1.29), (1.31), то справедливо 
СлЕДСТвИЕ 2.9. Пусть выполнены условия предложения 2.6, $n=2 u$

$$
\mathfrak{C}_{N}(\mathbf{F}, \mathbf{G}) \equiv(\mathbf{F}, 1)_{\mathbb{R}^{2} \backslash \omega}+(\mathbf{G}, 1)_{\partial \omega}=0 .
$$

Тогда существует единственное, исчезающее на бесконечности решение задачи (1.29), (1.31), и оно подчинено (2.11).

4. О логарифмической емкости множества $\omega(n=2)$. В этом и следуюших двух разделах для внешних задач (1.29), (1.30) и (1.29), (1.31) вводятся характеристики, аналогичные таким классическим объектам теории гармонических функций, как емкость и тензор присоединенной массы (см. [39] и др.). За ними сохраняются прежние названия, хотя замешение оператора Лапласа оператором $L_{0}\left(0, y, \partial_{y}\right)$ приводит не только к изменению названных интегральных характеристик множества $\omega$, но и к модификации самих определений. Используемые далее свойства этих характеристик приводятся без доказательств, поскольку их проверка следует стандартной схеме (см., например, [39]) с изменениями, вызванными периодичностью коэффициентов, но обсуждавшимися подробно в [31; §5].

Пусть $n=2$ и $\Psi(x)$ - фундаментальное решение главной (в точке $x=0$ ) части $\mathbf{L}^{0}\left(\partial_{y}\right)$ осредненного оператора (1.25),

$$
\Psi(y)=-\sigma \log \rho+\psi(\varphi)
$$

(оно уже возникало в (2.8)). Так как $\Psi$ определено с точностью до постоянного слагаемого, можно соблюсти условие $(\psi, 1)_{S^{1}}=0$. Рассмотрим сумму

$$
\mathscr{X}(y)=\Psi(y)+\sum_{j=1}^{n} Y_{j}(0, y) \frac{\partial \Psi}{\partial y_{j}}(y),
$$

в которой $Y_{j}$ - специальные решения ячеечного уравнения, фигурируюшие в (1.26). Непосредственные вычисления при учете (1.23) и (1.27) показывают, что $L_{0}\left(0, y, \partial_{y}\right) \mathscr{X}(y)$ - линейная комбинация с ограниченными коэффициентами вторых и третьих производных функции $\Psi$. Таким образом, записывая решение однородной задачи (1.29), (1.30) в виде

$$
\zeta(y)=\left[1-\chi\left(\frac{\rho}{R_{e}}\right)\right] \mathscr{X}(y)+\zeta_{0}(y)
$$

(где $\chi \in C_{0}^{\infty}(-2,2), \chi(t)=1$ при $|t|<1$, а $R_{e}$ определено в п. 3), получаем для $\zeta_{0}$ задачу с правыми частями, удовлетворяюшими условиям предложения $2.5,1$ ), т.е. находим $\zeta_{0} \in \mathscr{D}$. Ясно, что $\mathscr{X} \notin \mathscr{D}$ и потому $\zeta$ - нетривиальное решение однородной задачи $(1.29),(1.30)$.

Для $\zeta_{0}$ справедливы формулы типа (2.12) и (2.11) с $|\alpha| \geqslant 1$. В соответствии c (2.15)-(2.17) перепишем их так:

$$
\zeta(y)=\mathscr{X}(y)+C_{\omega}+\cdots=-\sigma \log r+\psi(\varphi)+C_{\omega}+\cdots
$$

(многоточием обозначаются несушественные для текущих рассмотрений слагаемые). 
В случае оператора Лапласа $\zeta$ носит название функции Грина с полюсом на бесконечности, а в подобном (2.18) разложении

$$
\zeta(y)=-\frac{1}{2 \pi} \log \left[\frac{\rho}{c_{\log }(\omega)}\right]+\cdots
$$

фигурирует логарифмическая емкость $c_{\log }(\omega)$ (см. [40]). По аналогии, величину $\exp \left(-\sigma^{-1} C_{\omega}\right)$, возникающую в согласии с (2.18), назовем логарифмической $L_{0}$-емкостью множества $\omega$.

ПРЕДЛОЖЕНИЕ 2.10. В условиях предложения 2.6, при $n=2$ существует единственное решение $w$ задачи (1.29), (1.30), подчиненное соотношениям (2.11), $|\alpha|>0$, и (2.12), причем

$$
c_{\omega}=\mathfrak{C}_{D}(\mathbf{F}, \mathbf{G}) \equiv(\mathbf{F}, \zeta)_{\mathbb{R}^{2} \backslash \omega}-\left(\mathbf{G}, N_{0}\left(0, y, \partial_{y}\right) \zeta\right)_{\partial \omega}
$$

Вывод подобных (2.19) интегральных представлений для коэффициентов в асимптотике содержится в работе $[31 ; \S 5]$. Напоследок сформулируем два утверждения, ставшие очевидными.

СЛЕДСТВИЕ 2.11. 1) $\mathfrak{C}_{D}(0,1)=1$.

2) Равенство $\mathfrak{C}_{D}(\mathbf{F}, \mathbf{G})=0$ является необходимым и достаточныц условием (при $n=2)$ существования исчезающего на бесконечности решения задачи (1.29), (1.30).

5. О емкости множества $\omega(n=3)$. Фундаментальное решение $\Psi$ для оператора $\mathbf{L}^{0}\left(\partial_{y}\right)$ в $\mathbb{R}^{3}$ оказывается обобщенно однородной функцией порядка -1 , т.е. $\Psi(y)=\rho^{-1} \Psi^{0}(\varphi)$. По $\Psi$ построим сумму $\mathscr{X}$ так, как это указано в (2.16). Обозначим через $X$ исчезающее на бесконечности решение задачи $(1.29),(1.30)$ с $\mathbf{F}=0$, $\mathbf{G}=1$. В силу предложения 2.6 для него справедливы формулы вида (2.11). Обсудим главный член асимптотического разложения на бесконечности. После вычислений при учете (1.26), (1.27) имеем

$$
\begin{aligned}
L_{0}\left(0, \cdot \partial_{y}\right) \mathscr{X} & =\mathbf{L}^{0}\left(\partial_{y}\right) \Psi-b_{i j} \frac{\partial^{2} \Psi}{\partial y_{i} \partial y_{j}}-l_{i j} Y_{p} \frac{\partial^{3} \Psi}{\partial y_{i} \partial y_{j} \partial y_{p}} \\
b_{i j} & =l_{i j}+l_{i p} \frac{\partial Y_{j}}{\partial y_{p}}+\frac{\partial}{\partial y_{p}}\left(l_{p j} Y_{i}\right)-\mathbf{l}_{i j}
\end{aligned}
$$

(здесь принято соглашение о суммировании по $i, j, p=1,2,3$ ). Первое слагаемое справа в (2.20) равно нулю на $\mathbb{R}^{3} \backslash \bar{\omega}$. Функции $(2.21)$ являются гладкими $y$-периодическими и обладают нулевым средним по $\mathbf{S}$; обозначим через $B_{i j}$ решение уравнения (1.22) с $h=-b_{i j}$. Теперь

$$
L_{0}\left(0, y, \partial_{y}\right)\left\{\mathscr{X}(y)+\sum_{i, j=1}^{3} B_{i j}(y) \frac{\partial^{2} \Psi}{\partial y_{i} \partial y_{j}}(y)\right\}=O\left(\rho^{-4}\right), \quad \rho \rightarrow \infty .
$$

Столь быстрое убывание на бесконечности выражения (2.22) наводит на мысль, что сумма из фигурных скобок, умноженная на какую-то постоянную, служит 
главным членом асимптотики решения $X$. Это наблюдение подтверждается процедурой построения асимптотики, описанной в [30], [31]. Именно, выполняются неравенства, согласующиеся с (2.11):

$$
\begin{gathered}
\left|X(y)-C_{\omega} \Psi(y)\right| \leqslant c_{\delta} \rho^{\delta-2}, \quad\left|\partial_{y} X(y)-C_{\omega} \partial_{y} \mathscr{X}(y)\right| \leqslant c_{\delta} \rho^{\delta-2} \\
\left|\partial_{y} \partial_{y}^{\alpha}\left(X(y)-C_{\omega} \mathscr{X}(y)\right)\right| \leqslant c_{\alpha} \rho^{\delta-2}
\end{gathered}
$$

здесь $C_{\omega}$ - постоянная, зависящая от множества $\omega$, а $\delta$ - произвольное число из $(0,1)$. Подчеркнем, что вычитаемые в $(2.23)$ максимально упрощены - лишние члены отнесены в остаток. Укороченная запись (ср. с (2.18)) имеет вид

$$
X(y)=C_{\omega} \Psi(y)+\cdots .
$$

В случае оператора Лапласа $\Psi(y)=(4 \pi \rho)^{-1}$ и $X(y)=\operatorname{cap}(\omega) \rho^{-1}+O\left(\rho^{-2}\right)$. Поэтому величину $4 \pi C_{\omega}$ естественно назвать $L_{0}$-емкостью множества $\omega$. Справедлива следуюшая

Лемма 2.12. $C_{\omega}>0$.

6. Внешняя задача Неймана $(n=2,3)$; матрица присоединенной массы. Пусть $n=3$ и $X$ - решение задачи (1.29), (1.31) с $\mathbf{F}=0$ и какой-нибудь правой частью $\mathbf{G}$. Тогда справедливы неравенства $(2.23)$; вычислим коэффициент $C_{\omega}$, который теперь зависит от $\mathbf{G}$. Поскольку $\zeta=1$ - решение однородной задачи, то аналогично (2.19)

$$
C_{\omega}=(\mathbf{G}, 1) \partial \omega
$$

При $n=2$ аналогичное скалярное произведение фигурировало в условиях (2.14).

В [39; приложение G] интегральные характеристики внешней задачи Неймана связьвались с решениями, имеюшими линейный рост при $\rho \rightarrow \infty$. Найдем подобные решения для уравнения с периодическими коэффициентами. Положим

$$
\zeta_{k}(y)=y_{k}+Y_{k}(y)+X_{k}(y), \quad k=1, \ldots, n,
$$

где $Y_{k}$ указаны в $(1.26),(1.27)$, а $X_{k}$ - решение задачи $(1.29),(1.31)$, в которой

$$
\mathbf{F}=0, \quad \mathbf{G}_{k}(y)=-\sum_{i=1}^{n} \nu_{i}(y)\left\{l_{i k}(y)+\sum_{j=1}^{n} l_{i j}(y) \frac{\partial Y_{k}}{\partial y_{j}}(y)\right\} .
$$

Так как $L_{0}\left(0, y, \partial_{y}\right)\left(y_{k}+Y_{k}(y)\right)=0$ и

$$
\left(\mathbf{G}_{k}, 1\right)_{\partial \omega}=-\int_{\omega} L_{0}\left(0, y, \partial_{y}\right)\left(y_{k}+Y_{k}(y)\right) d y=0
$$

то в силу утверждений $2.5,1), 2.9$ и 2.6 (а также согласно $(2.24)$ в случае $n=3$ ) решение $X_{k}(y)$ убьвает, как $O\left(\rho^{1-n}|\log \rho|^{s}\right)$. Примем во внимание член

$$
\rho^{1-n}\left[w^{(n-1)}(\varphi, \log \rho)+W^{(n-1)}(\varphi, \log \rho, y)\right]
$$


разложения $X_{k}(y)$. Если коэффициенты постоянны, то осциллирующая добавка $W^{(n-1)}$ отсутствует, и согласно [24], [25] $\rho^{n-1} w^{(n-1)}$ оказывается линейной комбинацией производных фундаментального решения $\Psi$. Этот же результат сохраняется и для уравнения с периодическими коэффициентами - со ссылками на статьи [30], [31] напишем аналогично (2.24):

$$
\left|X_{k}(y)-\sum_{p=1}^{n} m_{k p}^{\omega} \frac{\partial \Psi}{\partial y_{p}}(y)\right| \leqslant c \rho^{\delta-n}, \quad\left|\partial_{k} X_{k}(y)-\sum_{p=1}^{n} m_{k p}^{\omega} \frac{\partial \mathscr{X}_{k}}{\partial y_{p}}(y)\right| \leqslant c \rho^{\delta-n}
$$

здесь $m^{\omega}=\left\|m_{k p}^{\omega}\right\|$ - некоторая $(n \times n)$-матрица, зависящая от $\omega$, и

$$
\mathscr{X}_{k}=\frac{\partial \Psi}{\partial y_{k}}+\sum_{j=1}^{n} Y_{j} \frac{\partial^{2} \Psi}{\partial y_{j} \partial y_{k}}
$$

(ср. с (2.16)). В случае оператора Лапласа $m^{\omega}$ - матрица (тензор) присоединенной массы для множества $\omega$ (см. [39]). В рассматриваемом случае матрица $m^{\omega}$ сохраняет базовые свойства (см. очередную лемму), и потому мы не изменяем ее названия.

ЛЕмма 2.13. Матрица ${ }^{\omega}$ симметрическая и положсительно определенная.

ЗАмЕчАниЕ 2.14. В случае оператора Лапласа рассмотренные интегральные характеристики не зависят от положения множества $\omega$ относительно фиксированной системы декартовых координат (в частности, $m^{\omega}-$ тензор; см. [39]). Для опеpaтора $L_{0}\left(0, y, \partial_{y}\right)$ это не так - без каких-либо предположений о симметрии матрицы $\left\|l_{i j}(0, y)\right\|$ характеристики неизменны только при переносе начала координат из одного узла целочисленной решетки в другой при сохранении направлений осей (именно поэтому $m^{\omega}$ именуется у нас матрицей).

\section{§ 3. Построение асимптотического разложения решения трехмерной задачи Дирихле}

1. Старший член асимптотики. Случай $l_{0}>0$. Для построения старшего члена асимптотического разложения применим классическую процедуру осреднения [3]-[8].

ЛЕмма 3.1 (см., например, [5]). Пусть $\varphi(x, y)-y$-периодическая функиия из $C^{\infty}(\bar{\Omega} \times \mathbf{S})$. Справедливо соотношение

$$
\begin{aligned}
& \mathscr{L}\left(x, \varepsilon^{-1} x, \partial_{x}\right) \varphi\left(x, \varepsilon^{-1} x\right) \\
& =\varepsilon^{-2}\left\{\begin{array}{l}
L_{0}\left(x, y, \partial_{y}\right) \varphi(x, y) \\
\left.\quad+\varepsilon L_{1}\left(x, y, \partial_{x}, \partial_{y}\right) \varphi(x, y)+\varepsilon^{2} L_{2}\left(x, y, \partial_{x}\right) \varphi(x, y)\right\}\left.\right|_{y=\varepsilon^{-1} x}
\end{array}\right.
\end{aligned}
$$

в котором $L_{0}$ - оператор (1.23),

$$
\begin{gathered}
L_{2}\left(x, y, \partial_{x}\right)=-\sum_{i, j=1}^{n} \frac{\partial}{\partial x_{i}} l_{i j}(x, y) \frac{\partial}{\partial x_{j}}+l_{0}(x, y) \\
L_{1}\left(x, y, \partial_{x}, \partial_{y}\right)=-\sum_{i, j=1}^{n}\left(\frac{\partial}{\partial x_{i}} l_{i j}(x, y) \frac{\partial}{\partial y_{j}}+\frac{\partial}{\partial y_{i}} l_{i j}(x, y) \frac{\partial}{\partial x_{j}}\right) .
\end{gathered}
$$


Основной член асимптотики решения задачи $(1.2),(1.5),(1.6)$ вне любой фиксированной окрестности множества $\omega_{\varepsilon}$ представим как сумму

$$
U_{0}(x)+\varepsilon \sum_{k=1}^{3} Y_{k}(x, y) \frac{\partial U_{0}}{\partial x_{k}}(x)+\varepsilon^{2} v_{0}(x, y),
$$

где $y=\varepsilon^{-1} x$, а $v_{0}$ - некоторая гладкая $y$-периодическая функция с нулевьм средним по $\mathbf{S}$. Помещая (3.3) в (1.2), получаем для $v_{0}$ уравнение (1.22) с правой частью

$$
h_{U}(x, y)=f(0, x, y)-L_{1}\left(x, y, \partial_{x}, \partial_{y}\right) \sum_{k=1}^{3} Y_{k}(x, y) \frac{\partial U_{0}}{\partial x_{k}}(x)-L_{2}\left(x, y, \partial_{x}\right) U_{0}(x)
$$

(далее мы откажемся от такого определения $\left.v_{0}\right)$. В силу формул (1.25), (1.26) и (3.2) условие разрешимости названного уравнения принимает вид

$$
\mathbf{L}\left(x, \partial_{x}\right) U_{0}(x)=\left\langle f^{(0)}\right\rangle(x)
$$

при этом $f^{(0)}(x, y)=f(0, x, y)$, а обозначение $\left\langle f^{(0)}\right\rangle$ введено в (1.24). Таким образом, слагаемое $U_{0}$ суммы (3.3) определяется из задачи (3.5), (1.5).

Функция (3.3) не удовлетворяет краевому условию (1.6). Поправку типа пограничного слоя ищем в виде произведения

$$
\chi_{\Omega}(x) w_{0}\left(\varepsilon^{-1} x\right),
$$

где $\chi_{\Omega}(x)=\chi\left(d_{\Omega}|x| / 2\right)$, a $d_{\Omega}-$ радиус шара, содержашегося в $\Omega$; эталонная срезка $\chi$ введена после (2.17). Разложим коэффициенты оператора $\mathscr{L}$ в ряды Тейлора в окрестности точки $x=0$ и перейдем к быстрым переменньм $y=\varepsilon^{-1} x$; оператор $\mathscr{L}$ допускает расщепление

$$
\mathscr{L} \sim \varepsilon^{-2}\left\{M^{(0)}\left(y, \partial_{y}\right)+\varepsilon M^{(1)}\left(y, \partial_{y}\right)+\varepsilon^{2} M^{(2)}\left(y, \partial_{y}\right)+\cdots\right\},
$$

в котором $M^{(0)}\left(y, \partial_{y}\right)=L_{0}\left(0, y, \partial_{y}\right)($ см. $(1.23))$ и

$$
\begin{aligned}
M^{(k)}\left(y, \partial_{y}\right)= & -\left.\sum_{i, j=1}^{n} \frac{\partial}{\partial y_{i}} \sum_{|\alpha|=k} \frac{1}{\alpha !} y^{\alpha} \partial_{x}^{\alpha} l_{i j}(x, y)\right|_{x=0} \frac{\partial}{\partial y_{j}} \\
& +\left.\sum_{|\alpha|=k-2} \frac{1}{\alpha !} y^{\alpha} \partial_{x}^{\alpha} l_{0}(x, y)\right|_{x=0} .
\end{aligned}
$$

Здесь при $n=3$ использованы мультииндекс $\alpha=\left(\alpha_{1}, \ldots, \alpha_{n}\right)$ и обозначения

$$
y^{\alpha}=y_{1}^{\alpha_{1}} \cdots y_{n}^{\alpha_{n}}, \quad \partial_{x}^{\alpha}=\frac{\partial^{|\alpha|}}{\partial x_{1}^{\alpha_{1}} \cdots \partial x_{n}^{\alpha_{n}}}, \quad \alpha !=\alpha_{1} ! \cdots \alpha_{n} !
$$

Теперь ясно, что функция $w_{0}$ должна удовлетворять однородному уравнению (1.29), содержашему слева главную часть операторного ряда (3.7). Поскольку 
роль пограничного слоя заключается в компенсации невязки на $\partial \omega_{\varepsilon}$ асимптотического решения (3.3), естественно подчинить $w_{0}$ краевому условию $(1.30)$, в котором $\mathbf{G}(y)=-U_{0}(0)$. Таким образом,

$$
w_{0}(y)=-U_{0}(0) X(y),
$$

где $X$ - емкостный потенциал, введенный в п. $5 \oint 2$.

На первый взгляд кажется, что построение основного члена (3.3) завершено, однако быстрая осцилляция коэффициентов приводит к неожиданному эффекту: пограничный слой (3.6) привносит в (1.2) быстроосциллирующую невязку $O(1)$, которую следует компенсировать слагаемьм $v_{0}$ из (3.3). В самом деле, учитывая $(2.23)$ и $(3.8)$, напишем

$$
\begin{aligned}
\chi(x) w_{0}\left(\frac{x}{\varepsilon}\right) & =-\chi(x) U_{0}(0) c_{\omega}\left\{\Psi(y)+\sum_{p=1}^{3} Y_{p}(0, y) \frac{\partial \Psi}{\partial y_{p}}(y)\right\}+\cdots \\
& =-\chi(x) U_{0}(0) c_{\omega}\left\{\varepsilon \Psi(x)+\varepsilon^{2} \sum_{p=1}^{3} Y_{p}(0, y) \frac{\partial \Psi}{\partial x_{p}}(x)\right\}+\cdots
\end{aligned}
$$

применим лемму 3.1 и получим

$$
\begin{aligned}
\mathscr{L}\left(x, \frac{x}{\varepsilon}, \partial_{x}\right)\left(\chi(x) w_{0}\left(\frac{x}{\varepsilon}\right)\right) \\
=U_{0}(0) c_{\omega} \sum_{j, k=1}^{3}\left\{\frac{\partial}{\partial y_{j}} l_{j k}(x, y) \frac{\partial}{\partial x_{k}}(\chi(x) \Psi(x))\right. \\
\left.\quad+\chi(x) \frac{\partial}{\partial y_{j}} l_{j k}(x, y) \frac{\partial}{\partial y_{k}}\left(\sum_{p=1}^{3} Y_{p}(0, y) \frac{\partial \Psi}{\partial x_{p}}(x)\right)\right\}+\cdots
\end{aligned}
$$

Здесь многоточием обозначены члены, содержащие $\varepsilon^{h}$ с $h>0$. Даже в случае $\chi=1$ выражение из фигурных скобок в (3.9) аннулируется только при условии независимости коэффициентов $l_{j k}$ от медленных переменных $x$ (в соответствии с определением $Y_{p}$; см. (1.27)). Таким образом, функция (3.6) порождает дополнительную невязку

$$
\begin{aligned}
& h_{w}(x, y)=U_{0}(0) c_{\omega} \sum_{j, k=1}^{3}\left\{\frac{\partial l_{j k}}{\partial y_{j}}(x, y) \Psi(x) \frac{\partial \chi}{\partial x_{k}}(x)\right. \\
& \left.\quad+\chi(x) \frac{\partial}{\partial y_{j}}\left(l_{j k}(x, y)-l_{j k}(0, y)\right)\left(\frac{\partial \Psi}{\partial x_{k}}(x)+\sum_{p=1}^{3} \frac{\partial Y_{p}}{\partial y_{k}}(0, y) \frac{\partial \Psi}{\partial x_{p}}(x)\right)\right\} .
\end{aligned}
$$

Ясно, что $\left\langle h_{w}\right\rangle=0$, т.е. выражение (3.10) удается компенсировать при помощи слагаемого $v_{0}$ из (3.3), если определить его как решение уравнения $(1.22)$ с правой частью $h=h_{U}+h_{w}$. Соответственно, $v_{0}=v_{0 U}+v_{0 w}$, причем $v_{0 U}$ - гладкая функция обеих переменных $x$ и $y$, но ввиду присутствия в (3.10) фундаментального решения $\Psi(x)=O\left(r^{-1}\right)$ и разностей $l_{j k}(x, y)-l_{j k}(0, y)=O(r)$ функция $v_{0 w}$ удовлетворяет таким соотношениям:

$$
\left|\partial_{x}^{\alpha} \partial_{y}^{\sigma} v_{0 w}(x, y)\right| \leqslant c_{\alpha \sigma} r^{-1-|\alpha|} .
$$

В (3.11) $\alpha$ и $\sigma$ - произвольные мультииндексы, $r=|x|$. 


\section{2. Обоснование старшего члена асимптотики. Пусть}

$$
\begin{aligned}
\mathscr{V}_{0}(\varepsilon, x)= & \left(1-\chi_{\omega}\left(\frac{x}{\varepsilon}\right)\right)\left\{U_{0}(x)+\varepsilon \sum_{k=1}^{3} Y_{k}\left(x, \frac{x}{\varepsilon}\right) \frac{\partial U_{0}}{\partial x_{k}}(x)+\varepsilon^{2} v_{0}\left(x, \frac{x}{\varepsilon}\right)\right\} \\
& +\chi_{\omega}\left(\frac{x}{\varepsilon}\right) U_{0}(0)+\chi_{\Omega}(x) w_{0}\left(\frac{x}{\varepsilon}\right),
\end{aligned}
$$

где $\chi_{\Omega}$ - та же срезка, что и в $(3.6), \chi_{\omega}\left(\varepsilon^{-1} x\right)=\chi\left(\varepsilon^{-1} d_{\omega}|x|\right)$, а $d_{\omega}$ - радиус шара, содержашего $\bar{\omega}$. Разность $\mathscr{R}_{0}=u-\mathscr{V}_{0}$ удовлетворяет задаче

$$
\mathscr{L} \mathscr{R}_{0}=\mathscr{F}_{0} \quad \text { в } \Omega(\varepsilon), \quad \mathscr{R}_{0}=0 \text { на } \partial \omega_{\varepsilon}
$$

(для краткости условия периодичности (1.5) не пишем). Краевое условие в (3.13) получилось однородньм благодаря введению в $(3.12)$ срезаюшей функции $\chi_{\omega}$, поскольку в силу $(3.8),(3.11)$ и равенства $X=1$ на $\partial \omega$ имеем

$$
\mathscr{V}_{0}(\varepsilon, x)=0+U_{0}(0)+w_{0}\left(\varepsilon^{-1} x\right)=U_{0}(0)-U_{0}(0) \text { при } x \in \partial \omega_{\varepsilon} .
$$

Пользуясь соотношениями для функций $U_{0}, v_{0}, w_{0}$ и $Y_{k}$, справедливыми согласно их построению, представим правую часть $\mathscr{F}_{0}$ как сумму шести слагаемых:

$$
\begin{aligned}
& \mathscr{F}_{0}^{1}(\varepsilon, x)=-\varepsilon\left(1-\chi_{\omega}\left(\frac{x}{\varepsilon}\right)\right)\left\{L_{2}\left(x, y, \partial_{x}\right) \sum_{k=1}^{3} Y_{k}(x, y) \frac{\partial U_{0}}{\partial x_{k}}(x)\right. \\
& \left.+\left(L_{1}\left(x, y, \partial_{x}, \partial_{y}\right)+\varepsilon L_{2}\left(x, y, \partial_{x}\right)\right) v_{0}(x, y)\right\}, \\
& \mathscr{F}_{0}^{2}(\varepsilon, x)=f(\varepsilon, x, y)-\left(1-\chi_{\omega}\left(\varepsilon^{-1} x\right)\right) f(0, x, y) \text {, } \\
& \mathscr{F}_{0}^{3}(\varepsilon, x)=\left[\mathscr{L}\left(x, \varepsilon^{-1} x, \partial_{x}\right), \chi_{\omega}\left(\varepsilon^{-1} x\right)\right] \\
& \times\left(U_{0}(x)-U_{0}(0)+\varepsilon \sum_{k=1}^{3} Y_{k}(x, y) \frac{\partial U_{0}}{\partial x_{k}}(x)+\varepsilon^{2} v_{0}(x, y)\right), \\
& \mathscr{F}_{0}^{4}(\varepsilon, x)=-\chi_{\omega}\left(\varepsilon^{-1} x\right) l_{0}\left(x, \varepsilon^{-1} x\right) U_{0}(0), \\
& \mathscr{F}_{0}^{5}(\varepsilon, x)=-\left[\mathscr{L}\left(x, \varepsilon^{-1} x, \partial_{x}\right), \chi_{\Omega}(x)\right] w_{0}\left(\varepsilon^{-1} x\right) \\
& -U_{0}(0) c_{\omega}\left[L_{1}\left(x, y, \partial_{x}, \partial_{y}\right), \chi_{\Omega}(x)\right] \Psi(x), \\
& \mathscr{F}_{0}^{6}(\varepsilon, x)=-\chi_{\Omega}(x)\left\{\left(\mathscr{L}\left(x, \varepsilon^{-1} x, \partial_{x}\right)-\mathscr{L}\left(0, \varepsilon^{-1} x, \partial_{x}\right)\right) w_{0}\left(\varepsilon^{-1} x\right)\right. \\
& +c_{\omega} U_{0}(0)\left(L_{1}\left(x, y, \partial_{x}, \partial_{y}\right)-L_{1}\left(0, y, \partial_{x}, \partial_{y}\right)\right) \Psi(x) \\
& \left.+c_{\omega} U_{0}(0)\left(L_{0}\left(x, y, \partial_{y}\right)-L_{0}\left(0, y, \partial_{y}\right)\right) \sum_{p=1}^{3} Y_{p}(0, y) \frac{\partial \Psi}{\partial x_{p}}(x)\right\} \text {. }
\end{aligned}
$$

Отметим, что при формировании $\mathscr{F}_{0}^{5}$ и $\mathscr{F}_{0}^{6}$ принято во внимание выражение (3.10).

ПРЕДЛОЖЕНИЕ 3.2. При условии (1.8) имеет место оценка

$$
\left\|\mathscr{R}_{0} ; H^{1}(\Omega(\varepsilon))\right\| \leqslant c_{\delta} \varepsilon^{1-\delta},
$$

где $\delta$ - произвольное положительное число, но постоянная $c_{\delta}$ мохет завиcemь om $\delta$. 
ДоКАЗАТЕЛЬСТВо. Вспоминая соотношения (1.11), (1.12) и (2.5), получаем неравенство

$$
c_{0}\left\|\mathscr{R}_{0} ; H^{1}(\Omega(\varepsilon))\right\| \leqslant\left\|r \mathscr{F}_{0} ; L_{2}(\Omega(\varepsilon))\right\| \equiv\left|\mathscr{F}_{0}\right| .
$$

Оценим правую часть, основываясь на (3.14). Поскольку $|f(\varepsilon, x, y)-f(0, x, y)| \leqslant$ $c \varepsilon$, имеем

$$
\left|\mathscr{F}_{0}^{2}\right|^{2} \leqslant c\left(\varepsilon^{2}+\int_{\left\{r<d_{\omega} \varepsilon\right\}} r^{2}|f(0, x, y)|^{2} d x\right) \leqslant c\left(\varepsilon^{2}+\varepsilon^{5}\right) .
$$

Столь же очевидно, что $\left|\mathscr{F}_{0}^{4}\right|^{2} \leqslant c \varepsilon^{5}$.

Ввиду формулы (3.11) и гладкости функций $U_{0}, v_{0 U}$ выводим для $\mathscr{F}_{0}^{1}$ следуюшее неравенство:

$$
\left|\mathscr{F}_{0}^{1}\right|^{2} \leqslant c \varepsilon^{2} \int_{\Omega(\varepsilon)} r^{2}\left(1+r^{-4}+\varepsilon^{2} r^{-6}\right) d x \leqslant c \varepsilon^{2} .
$$

Так как $U_{0}(x)-U_{0}(0)=O(r)$, то по тем же причинам

$$
\left|\mathscr{F}_{0}^{3}\right|^{2} \leqslant c \varepsilon^{-4} \int_{\left\{r<2 d_{\omega} \varepsilon\right\}} r^{2}\left(r^{2}+\varepsilon^{2}+\varepsilon^{4}\left(1+r^{-2}\right)\right) d x \leqslant c \varepsilon^{3} .
$$

Для обработки $\mathscr{F}_{0}^{5}$ и $\mathscr{F}_{0}^{6}$ вспомним асимптотику емкостного потенциала $X$, построенного в п. $5 \S 2$. В силу (3.1) [ $\left.\mathscr{L}, \chi_{\Omega}\right]=\left[\varepsilon^{-1} L_{1}+L_{2}, \chi_{\Omega}\right]-$ дифференциальный оператор с коэффициентами, равными нулю при $r<d_{\Omega} / 2$. Пользуясь оценками $(2.23)$ с $|\alpha|=0$ и замечая, что слагаемое $\varepsilon^{-1}\left[L_{1}, \chi_{\Omega}\right]\left(\varepsilon U_{0}(0) c_{\omega} \Psi\right)$ удалено из $\left[\mathscr{L}, \chi_{\Omega}\right] w_{0}$, приходим к неравенству

$$
\left|\mathscr{F}_{0}^{5}\right|^{2} \leqslant c \int_{\Omega(\varepsilon)} r^{2}\left\{\varepsilon^{-2}\left(\frac{r}{\varepsilon}\right)^{2 \delta-4}+\left(\frac{r}{\varepsilon}\right)^{2}\right\} d x \leqslant c_{\delta} \varepsilon^{2(1-\delta)} .
$$

Теперь разберемся с $\mathscr{F}_{0}^{6}$. Представим $w_{0}$ в виде

$$
\begin{aligned}
w_{0} & =K\left(\Psi(y)+\sum_{p=1}^{3} Y_{p}(0, y) \frac{\partial \Psi}{\partial y_{p}}(y)\right)+\widetilde{w}_{0}(y) \\
& =K\left(\varepsilon \Psi(x)+\varepsilon^{2} \sum_{p=1}^{3} Y_{p}(0, y) \frac{\partial \Psi}{\partial x_{p}}(x)\right)+\widetilde{w}_{0}(y),
\end{aligned}
$$

где $K=-U_{0}(0) c_{\omega}$. Ссылаясь на лемму 3.1 , находим

$$
\begin{aligned}
\mathscr{L}\left(x, \varepsilon^{-1} x, \partial_{x}\right) w_{0}\left(\varepsilon^{-1} x\right) & =\mathscr{L}\left(x, \varepsilon^{-1} x, \partial_{x}\right) \widetilde{w}_{0}\left(\varepsilon^{-1} x\right) \\
+ & K\left(L_{1}\left(x, y, \partial_{x}, \partial_{y}\right)+\varepsilon L_{2}\left(x, y, \partial_{x}\right)\right) \Psi(x) \\
+ & K\left(L_{0}\left(x, y, \partial_{y}\right)+\varepsilon L_{1}\left(x, y, \partial_{x}, \partial_{y}\right)+\varepsilon^{2} L_{2}\left(x, y, \partial_{x}\right)\right) \sum_{p=1}^{3} Y_{p}(0, y) \frac{\partial \Psi}{\partial x_{p}}(x) .
\end{aligned}
$$

По сравнению с этой формулой в представлении $\mathscr{F}_{0}^{6}$ (см. (3.14)) фигурируют разности операторов, коэффициенты которых суть $O(r)$ (поэтому в очередном интегральном неравенстве возникает дополнительный весовой множитель $r^{2}$ ). Члены, 
не имеющие коэффициентов $\varepsilon$ или $\varepsilon^{2}$, устраняются вычитанием, а норма слагаемого, содержашего $\widetilde{w}_{0}$, оценивается при помощи (2.23) с мальм $\delta$. Учитывая соотношения $\partial_{x}^{\alpha} \Psi(x)=O\left(r^{-1-|\alpha|}\right)$, заключаем, что

$$
\left|\mathscr{F}_{0}^{6}\right|^{2} \leqslant c \int_{\Omega(\varepsilon)} r^{2} \cdot r^{2}\left\{\left(\frac{r}{\varepsilon}\right)^{2 \delta-4}+\varepsilon^{2} r^{-6}+\varepsilon^{2} r^{-6}+\varepsilon^{4} r^{-8}\right\} d x \leqslant c \varepsilon^{2},
$$

где $\delta>0$. Осталось собрать приведенные формулы.

Упростим асимптотическое приближение (3.12), удалив из него срезающие функции, которыенужны были только для соблюдения краевого условия (1.6). Именно, положим

$$
\mathscr{U}_{0}(\varepsilon, x)=U_{0}(x)+\varepsilon \sum_{k=1}^{3} Y_{k}\left(x, \frac{x}{\varepsilon}\right) \frac{\partial U_{0}}{\partial x_{k}}(x)+\varepsilon^{2} v_{0}\left(x, \frac{x}{\varepsilon}\right)-\chi_{\Omega} U_{0}(0) X\left(\frac{x}{\varepsilon}\right) .
$$

Непосредственными вычислениями убеждаемся в том, что

$$
\left\|\mathscr{U}_{0}-\mathscr{V}_{0} ; H^{1}(\Omega(\varepsilon))\right\| \leqslant c \varepsilon
$$

Таким образом, в силу (3.15), (3.17) и (2.5) справедливо следующее утверждение.

Теорема 3.3. При условии (1.8) решение задачи (1.2), (1.5), (1.6) и его асимптотическое приближение (3.16) связань неравенством

$$
\begin{aligned}
\left\|u-\mathscr{U}_{0}\right\| & \equiv\left\|r^{-1}\left(u-\mathscr{U}_{0}\right) ; L_{2}(\Omega(\varepsilon))\right\|+\left\|\partial_{x}\left(u-\mathscr{U}_{0}\right) ; L_{2}(\Omega(\varepsilon))\right\| \\
& \leqslant c\left\|u-\mathscr{U}_{0} ; H^{1}(\Omega(\varepsilon))\right\| \leqslant c_{\delta} \varepsilon^{1-\delta},
\end{aligned}
$$

где $\delta>0$ - произвольное число, а постоянная $c_{\delta}$ не зависит от $\varepsilon \in\left(0, \varepsilon_{0}\right]$.

Итак, главный член асимптотики решения задачи (1.2), (1.5), (1.6) содержит две асимптотические поправки - быстроосциллирующую и типа пограничного слоя около $\partial \omega_{\varepsilon}$. Согласно $(2.12)$ на удалении от $\bar{\omega}_{\varepsilon}$ обе поправки имеют порядок $\varepsilon$, но вблизи отверстия превалирует $U_{0}(0) X$. В формулах для производных решения главенствует пограничный слой.

При помоши (3.11) и (2.23) находим, что

$$
\begin{gathered}
\left\|\varepsilon^{2} v_{0} ; H^{1}(\Omega(\varepsilon))\right\| \leqslant c \varepsilon^{2}\left(\int_{\varepsilon}^{c}\left[r^{-1}\left(r^{-1}+\varepsilon^{-1}\right)\right]^{2} r^{2} d r\right)^{1 / 2} \leqslant c \varepsilon, \\
\left\|\chi_{\Omega} U_{0}(0) X ; H^{1}(\Omega(\varepsilon))\right\| \leqslant c\left(\int_{\varepsilon}^{c}\left[\varepsilon^{-1}\left(1+\frac{r}{\varepsilon}\right)^{-2}\right]^{2} r^{2} d r\right)^{1 / 2} \leqslant c \varepsilon^{1 / 2} .
\end{gathered}
$$

Значит, слагаемое $\varepsilon^{2} v_{0}(x, y)$ устраняется из асимптотического приближения (3.16) безболезненно - соотношение (3.18) сохраняется. В то же время удаление члена $-\chi_{\Omega}(x) U_{0}(0) X\left(\varepsilon^{-1} x\right)$, описывающего пограничный слой, приводит к потере точности:

$$
\left\|u-\left(U_{0}+\varepsilon \sum_{k=1}^{3} Y_{k} \frac{\partial U_{0}}{\partial x_{k}}\right)\right\| \leqslant c \varepsilon^{1 / 2} .
$$


Подчеркнем, что именно такая мажоранта $c \varepsilon^{1 / 2}$ получается обычно (см. [3]-[8] и др.) при оправдании процедуры осреднения без учета каких-либо пограничных слоев. Убрать корректоры $Y_{k}$ из последней формулы нельзя, так как их норма в $H^{1}(\Omega(\varepsilon))$ составляет $O(1)$.

Теорема 3.3 дает оценку разности $u-\mathscr{U}_{0}$ по энергетической метрике. Вместе с тем, применяя принцип максимума и используя прежнее представление (3.14) правой части задачи (3.13), можно установить такое неравенство:

$$
\begin{aligned}
\mid u(\varepsilon, x)-\left\{U_{0}(x)\right. & +\varepsilon \sum_{k=1}^{3} Y_{k}\left(x, \frac{x}{\varepsilon}\right) \frac{\partial U_{0}}{\partial x_{k}}(x) \\
& \left.+\varepsilon^{2} v_{0}\left(x, \frac{x}{\varepsilon}\right)-\chi_{\Omega} U_{0}(0) X\left(\frac{x}{\varepsilon}\right)\right\} \mid \leqslant c_{\delta} \varepsilon^{1-\delta} \quad \text { при } \quad x \in \overline{\Omega(\varepsilon)} .
\end{aligned}
$$

Теперь без изменения мажоранты $O\left(\varepsilon^{1-\delta}\right)$ из фигурных скобок можно удалить как $\varepsilon Y_{k} \frac{\partial U_{0}}{\partial x_{k}}$, так и $\varepsilon^{2} v_{0}$ (напоминаем, что согласно $(3.11) \varepsilon^{2} v_{0}\left(x, \varepsilon^{-1} x\right)=O(\varepsilon)$ при $x \in \Omega(\varepsilon))$, однако поправка типа пограничного слоя обязана оставаться. Впрочем, в соответствии с (2.23) можно обойтись без срезающей функции $\chi_{\Omega}$ и упростить формулу до такой:

$$
\left|u(\varepsilon, x)-\left\{U_{0}(x)-U_{0}(0) X\left(\frac{x}{\varepsilon}\right)\right\}\right| \leqslant C_{\delta} \varepsilon^{1-\delta} \quad \text { при } \quad x \in \overline{\Omega(\varepsilon)}
$$

3. Построение полной асимптотики. Как уже говорилось, нам придется пользоваться процедурой перераспределения невязок. Дело в том, что коэффициенты операторов $M^{(p)}$ суть полиномы от $y$, и потому при использовании обычных итерационных процедур в правых частях уравнений (1.29) для $w_{k}$ окажутся слагаемые $O\left(\rho^{-j+k}\right)$, где $j, k=1,2, \ldots$ Это означает, что $w_{k}$ обязательно содержат выражения $O\left(\rho^{2-j+k}\right)$ и, тем самым, теряют основное свойство пограничного слоя - затухание на бесконечности. Для устранения несоответствия изменим процедуру и сохраним справа в (1.29) лишш остаток $o\left(\rho^{-2}\right)$, отшепив члены $O\left(\rho^{k}\right)$ с $k \geqslant-2$, пригодные для уравнений (1.22) и (1.28) после замены $\rho \mapsto r$. Иными словами, невязка, образованная $w_{k}$, компенсируется не только решениями $w_{k+1}, w_{k+2}, \ldots$ типа пограничного слоя, но и решениями $U_{k+1}, v_{k+1}, U_{k+2}, v_{k+2}, \ldots$ других типов. Аналогично, неудобные части невязок, порожденных $U_{k}$ и $v_{k}$, после замены $r \mapsto \rho$ учитываются при решении внешних задач для $w_{k+1}, w_{k+2}, \ldots$ В такой комбинированной обработке всех возникающих невязок и состоит процедура их перераспределения. Реализуем ее в рассматриваемой ситуации.

Предположим, что при $0 \leqslant j<k$ функции $U_{j}, v_{j}$ и $w_{j}$, допускающие соответственно представления $(2.2),(2.4)$ с $\mu=-2$ и (2.11), построены. Найдем $U_{k}, v_{k}$ и $w_{k}$.

Образованная функциями $v_{m}(m<k)$ невязка $L_{2} v_{k-2}+L_{1} v_{k-1}$ содержит слагаемые порядков $r^{-3}$ и $r^{-4}$; отшепим их и отправим в задачи для пограничного слоя. В правых частях уравнений $(1.22),(1.28)$ для определения $U_{k}, v_{k}$ останется 
выражение

$$
\begin{aligned}
F_{k}^{v}(x, y)= & -\sum_{q=1}^{2}\left\{L_{q}\left(x, y, \partial_{x}, \partial_{y}\right) v_{k-q}(x, y)\right. \\
& \left.-\chi_{\Omega}(x) \sum_{(p, m) \in S_{q}} L_{q}^{(p)}\left(x, y, \partial_{x}, \partial_{y}\right)\left(r^{-m} v_{k-q}^{(-m)}(\varphi, \log r, y)\right)\right\} .
\end{aligned}
$$

Здесь множество суммирования имеет вид

$$
S_{q}=\{(p, m): p=0,1, \ldots, q-1 ; m=3-q, \ldots, 2 ; p-m<0\},
$$

$v_{m}^{(j)}-$ коэффиициент разложения (2.4) функций $v_{k}$,

$$
\begin{aligned}
L_{2}^{(p)}\left(x, y, \partial_{x}\right)=- & \left.\sum_{i, j=1}^{3} \frac{\partial}{\partial x_{i}} \sum_{|\alpha|=p} \frac{1}{\alpha !} y^{\alpha} \partial_{x}^{\alpha} l_{i j}(x, y)\right|_{x=0} \frac{\partial}{\partial x_{j}} \\
& +\left.\sum_{|\alpha|=p-2} \frac{1}{\alpha !} x^{\alpha} \partial_{x}^{\alpha} l_{0}(x, y)\right|_{x=0} \\
L_{1}^{(p)}\left(x, y, \partial_{x}, \partial_{y}\right)=- & \sum_{i, j=1}^{3}\left\{\left.\frac{\partial}{\partial x_{i}} \sum_{|\alpha|=p} \frac{1}{\alpha !} y^{\alpha} \partial_{x}^{\alpha} l_{i j}(x, y)\right|_{x=0} \frac{\partial}{\partial y_{j}}\right. \\
& \left.+\left.\frac{\partial}{\partial y_{i}} \sum_{|\alpha|=p} \frac{1}{\alpha !} y^{\alpha} \partial_{x}^{\alpha} l_{i j}(x, y)\right|_{x=0} \frac{\partial}{\partial x_{j}}\right\}
\end{aligned}
$$

Функцию $F_{k}^{v}-\left\langle F_{k}^{v}\right\rangle$, обладаюшую нулевым средним по $\mathbf{S}$, поместим в уравнение $(1.22), \mathrm{a}\left\langle F_{k}^{v}\right\rangle-\mathrm{B}(1.28)$.

Аналогично поступим с коэффициентами $U_{m}(m<k)$ ряда (1.32). Допустимое в (1.28) слагаемое соответствующей невязки, попадающее в уравнение для $U_{k}$, имеет вид $\left\langle F_{k}^{U}\right\rangle$, где

$$
\begin{aligned}
& F_{k}^{U}(x, y)=-L_{2}\left(x, y, \partial_{x}\right) \sum_{j=1}^{3} Y_{j}(x, y) \frac{\partial U_{k-2}}{\partial x_{j}}(x) \\
& \quad+\chi_{\Omega}(x) L_{2}\left(0, y, \partial_{x}\right) \sum_{j=1}^{3} Y_{j}(0, y) \frac{\partial U_{k-2}^{(0)}}{\partial x_{j}}(\varphi, \log r)
\end{aligned}
$$

а $U_{m}^{(0)}-$ коэффициент представления $(2.2)$ функции $U_{m}$. Функция

$$
\left\langle F_{k}^{U}+h_{k}^{U}\right\rangle_{\perp}
$$

предназначена для нахождения $v_{k} . \mathrm{B}(3.19)$ и далее $\langle A\rangle_{\perp}=A-\langle A\rangle$. Слагаемое $h_{k}^{U}$ суммы (3.19) определяется по формуле

$$
h_{k}^{U}(x, y)=-L_{2}\left(x, y, \partial_{x}\right) U_{k}(x)-L_{1}\left(x, y, \partial_{x}, \partial_{y}\right) \sum_{j=1}^{3} Y_{j}(x, y) \frac{\partial U_{k-1}}{\partial x_{j}}(x) .
$$


В правых частях задач для нахождения $v_{k}$ и $U_{k}$ появляется невязка, образованная слагаемьми $\varepsilon^{m} w_{m}(m<k)$ пограничного слоя. Уравнение $(1.28)$ содержит среднее $\left\langle F_{k}^{w}\right\rangle$ функции

$$
\begin{aligned}
F_{k}^{w}(x, y)= & -\sum_{q=1}^{2} \sum_{(i, j) \in T_{k q}}\left\{\chi_{\Omega}(x)\left(L_{q}\left(x, y, \partial_{x}, \partial_{y}\right)-\sum_{p=0}^{j+q-3} L_{q}^{(p)}\left(x, y, \partial_{x}, \partial_{y}\right)\right)\right. \\
& \left.+\left[L_{q}\left(x, y, \partial_{x}, \partial_{y}\right), \chi_{\Omega}(x)\right]\right\}\left(r^{-j}\left(W_{i}^{(j)}(\varphi, \log r, y)+w_{i}^{(j)}(\varphi, \log r)\right)\right)
\end{aligned}
$$

при этом $T_{k q}=\{(i, j): i=0,1, \ldots ; j=1,2, \ldots ; i+j=k+2-q\}$.

Невязка, порожденная членами пограничного слоя и попадающая в правую часть (1.22), представима так:

$$
\left\langle F_{k}^{w}+h_{k}^{w}\right\rangle_{\perp}
$$

Помимо уже известной функции $F_{k}^{w}$ здесь фигурирует величина

$$
h_{k}^{w}(x, y)=-\chi_{\Omega}(x) \sum_{(p, q) \in T_{k 0}}\left(L_{0}\left(x, y, \partial_{y}\right)-\sum_{m=0}^{q-3} L_{0}^{(m)}\left(x, y, \partial_{y}\right)\right)\left(r^{-q} W_{p}^{(q)}(\varphi, \log r, y)\right)
$$

где

$$
L_{0}^{(p)}\left(x, y, \partial_{y}\right)=-\left.\sum_{i, j=1}^{3} \frac{\partial}{\partial y_{i}} \sum_{|\alpha|=p} \frac{1}{\alpha !} y^{\alpha} \partial_{x}^{\alpha} l_{i j}(x, y)\right|_{x=0} \frac{\partial}{\partial y_{j}}
$$

Итак, коэффициент $U_{k}$ асимптотического ряда (1.32) есть решение задачи (1.28), в которой

$$
F_{k}=\left\langle F_{k}^{v}+F_{k}^{U}+F_{k}^{w}+f_{k}\right\rangle_{\perp}
$$

a $f_{k}(x, y)$ - коэффициент ряда Маклорена для $f: \varepsilon \mapsto f(\varepsilon, x, y)$,

$$
f_{k}(x, y)=\frac{1}{k !} \frac{\partial^{k} f}{\partial \varepsilon^{k}}(0, x, y)
$$

Функция $v_{k}$ отыскивается из уравнения (1.22) с правой частью

$$
h_{k}=\left\langle F_{k}^{v}+F_{k}^{U}+F_{k}^{w}+h_{k}^{U}+h_{k}^{w}+f_{k}\right\rangle_{\perp} .
$$

Для описания задач, определяющих $w_{k}$, введем следующие обозначения. Пусть $\Upsilon(z, y)-y$-периодическая функция, принадлежащая $C^{\infty}\left(\mathbb{R}^{n} \times \mathbb{R}^{n}\right)$; тогда

$$
\frac{\partial}{\partial y_{j}} \Upsilon(y, y)=D_{j} \Upsilon(y)+\mathfrak{D}_{j} \Upsilon(y)
$$

где

$$
D_{j} \Upsilon(y)=\left.\frac{\partial \Upsilon}{\partial z_{j}}(z, y)\right|_{z=y}, \quad \mathfrak{D}_{j} \Upsilon(y)=\left.\frac{\partial \Upsilon}{\partial y_{j}}(z, y)\right|_{z=y}
$$


В уравнение (1.29) для $w_{k}$ поместим невязки $\mathbf{F}_{k}^{U}, \mathbf{F}_{k}^{v}$, образованные $U_{m}$ и $v_{m}$, $m \leqslant k$, и имеющие недопустимый для уравнений (1.22), (1.28) рост при $r \rightarrow 0$. Функция $\mathbf{F}_{k}^{U}$ имеет вид

$$
\mathbf{F}_{k}^{U}(x, y)=-M^{0,2}(y, D) \sum_{j=1}^{3} Y_{j}(0, y) \frac{\partial U_{k-1}^{(0)}}{\partial y_{j}}(\varphi, \log \rho)
$$

причем оператор $M^{0,2}(y, D)$ определяется равенством

$$
M^{k, 2}(y, D)=-\left.\sum_{i, j=1}^{n} D_{i} \sum_{|\alpha|=k} \frac{1}{\alpha !} y^{\alpha} \partial_{x}^{\alpha} l_{i j}(x, y)\right|_{x=0} D_{j}+\left.\sum_{|\alpha|=k-2} \frac{1}{\alpha !} y^{\alpha} \partial_{x}^{\alpha} l_{0}(x, y)\right|_{x=0}
$$

при $k=0$. Кроме того,

$$
\begin{aligned}
\mathbf{F}_{k}^{v}(x, y)= & -\sum_{(p, q, j) \in P_{k}} M^{q, 2}(y, D)\left(\rho^{j} v_{p}^{(j)}(\varphi, \log r, y)\right) \\
& +M^{0,1}(y, D, \mathfrak{D})\left(\rho^{-2} v_{k-2}^{(-2)}(\varphi, \log \rho, y)\right)
\end{aligned}
$$

здесь $P_{k}=\{(p, q, j): p, q=0,1, \ldots ; j=-2,-1, \ldots ; q+j<0 ; q+j+p=k-2\}$,

$$
\begin{aligned}
M^{k, 1}(y, D, \mathfrak{D})=-\sum_{i, j=1}^{n}\left\{D_{i} \sum_{|\alpha|=k}\right. & \left.\frac{1}{\alpha !} y^{\alpha} \partial_{x}^{\alpha} l_{i j}(x, y)\right|_{x=0} \mathfrak{D}_{j} \\
& \left.+\left.\mathfrak{D}_{i} \sum_{|\alpha|=k} \frac{1}{\alpha !} y^{\alpha} \partial_{x}^{\alpha} l_{i j}(x, y)\right|_{x=0} D_{j}\right\} .
\end{aligned}
$$

Итак, правая часть $\mathbf{F}_{k}$ уравнения (1.29) для $w_{k}$ равна сумме

$$
\mathbf{F}_{k}^{v}(x, y)+\mathbf{F}_{k}^{U}(x, y)+\mathbf{F}_{k}^{w}(x, y) .
$$

Здесь через $\mathbf{F}_{k}^{w}$ обозначена часть невязки, образованной членами $w_{m}, m<k$, пограничного слоя, исчезающая при $\rho \rightarrow+\infty$ как $o\left(\rho^{-2}\right)$. Именно,

$$
\begin{aligned}
\mathbf{F}_{k}^{w}(x, y)= & -\sum_{p=1}^{k}\left(M^{(p)}\left(y, \partial_{y}\right) w_{k-p}(y)-\sum_{m=0}^{2} M^{p, m}(y, D, \mathfrak{D})\right) \\
& \times\left(\sum_{j=1}^{p-m+2} \rho^{-j}\left(W_{k-p}^{(j)}(\varphi, \log \rho, y)+w_{k-p}^{(j)}(\varphi, \log \rho)\right)\right),
\end{aligned}
$$

где

$$
M^{k, 0}(y, \mathfrak{D})=-\left.\sum_{i, j=1}^{n} \mathfrak{D}_{i} \sum_{|\alpha|=k} \frac{1}{\alpha !} y^{\alpha} \partial_{x}^{\alpha} l_{i j}(x, y)\right|_{x=0} \mathfrak{D}_{j} .
$$

На этом процедура перераспределения невязок заканчивается - правая часть $\mathbf{G}_{k}$ краевого условия (1.30) на компакте $\partial \omega$ находится без ее участия. Используя 
разложения функций $U_{p}, Y_{j}$ и $v_{q}$ в ряды по степеням $r$, получаем следуюшее выражение для $G_{k}=\left.w_{k}\right|_{\partial \omega}$ :

$$
\begin{aligned}
\mathbf{G}_{k}(y)= & -\sum_{q=0}^{k} \rho^{q} U_{k-q}^{(q)}(\varphi, \log \rho)-\sum_{q=-2}^{k} \rho^{q} v_{k-q-2}^{(q)}(0, \varphi, y) \\
& -\left.\sum_{(p, q, m) \in Q_{k}} \sum_{j=1}^{3} \sum_{|\alpha|=p} \frac{1}{\alpha !} y^{\alpha} \partial_{x}^{\alpha} Y_{j}(x, y)\right|_{x=0}, \quad y \in \partial \omega ;
\end{aligned}
$$

здесь $Q_{k}=\{(p, q, m): p, q, m=0,1, \ldots ; p+q+m=k-1\}$.

Перейдем к оправданию асимптотики. Построим частичную сумму ряда (1.32)

$$
\begin{aligned}
\mathscr{U}_{N}(\varepsilon, x)=\sum_{k=0}^{N} \varepsilon^{k}\left\{U_{k}(x,\right. & \log \varepsilon)+\varepsilon \sum_{j=1}^{3} Y_{j}(x, y) \frac{\partial U_{k}}{\partial x_{j}}(x, \log \varepsilon) \\
& \left.+\varepsilon^{2} v_{k}(x, y, \log \varepsilon)+\chi_{\Omega}(x) w_{k}(x, y, \log \varepsilon)\right\}
\end{aligned}
$$

коэффициенты $U_{k}, v_{k}$ и $w_{k}$ которого найдены из задач $(1.28),(1.22)$ и (1.29), (1.30) с правыми частями, определенными по формулам (3.20), (3.21), (3.22). Невязки, образованные слагаемым $\chi_{\Omega} w_{k}$, не участвуют в определении $(3.20)$ функции $F_{k}$, однако входят в правые части равенства (3.21) для $h_{k}$. Поэтому порядок определения слагаемых суммы (3.25) с одинаковыми номерами таков: сначала отыскивается $U_{k}$, затем $w_{k}$ и, наконец, $v_{k}$.

$\mathrm{C}$ целью оценить погрешность приближения решения $u(\varepsilon, x)$ асимптотическим $\mathscr{U}_{N}(\varepsilon, x)$ поступаем так же, как и в п. 2. Сначала нужно построить вспомогательную функцию $\mathscr{V}_{N}$, полностью удовлетворяющую условию Дирихле (1.6) и мало отличающуюся от $\mathscr{U}_{N}$. Затем $\mathscr{V}_{N}$ подставляется в уравнения $(1.2),(1.6)$ и формируется задача

$$
\mathscr{L} \mathscr{R}_{N}=\mathscr{F}_{N} \quad \text { в } \Omega(\varepsilon), \quad \mathscr{R}_{N}=0 \quad \text { на } \partial \omega_{\varepsilon}
$$

для $\mathscr{R}_{N}=u-\mathscr{V}_{N}$. Остается оценить норму $\left|\mathscr{F}_{N}\right|$ и вывести подобное $(3.15)$ неравенство

$$
\left\|\mathscr{R}_{N} ; H^{1}(\Omega(\varepsilon))\right\| \leqslant c_{\delta} \varepsilon^{N+1-\delta}
$$

которое вместе с аналогичньм (3.17) соотношением

$$
\left\|\mathscr{U}_{N}-\mathscr{V}_{N} ; H^{1}(\Omega(\varepsilon))\right\| \leqslant c_{\delta} \varepsilon^{N+1}
$$

получающимся прямым вычислением нормы упомянутого продолжения, приводит к желанному результату. Самый трудоемкий, но в то же время рутинньй этап обработка правой части $\mathscr{F}_{N}$. Все особенности этого этапа, обусловленные соединением быстрой осцилляции коэффициентов и сингулярньм возмущением границы, были продемонстрированы достаточно подробно в п. 2. Поэтому здесь мы ограничиваемся формулировкой финальной оценки. 
Теорема 3.4. При условии (1.8) решение задачи (1.2), (1.5), (1.6) и его асимптотическое приближение (3.25) связаны неравенством

$$
\begin{aligned}
\left\|r^{-1}\left(u-\mathscr{U}_{N}\right) ; L_{2}(\Omega(\varepsilon))\right\| & +\left\|\nabla\left(u-\mathscr{U}_{N}\right) ; L_{2}(\Omega(\varepsilon))\right\| \\
& \leqslant c\left\|u-\mathscr{U}_{N} ; H^{1}(\Omega(\varepsilon))\right\| \leqslant c_{\delta} \varepsilon^{N+1-\delta},
\end{aligned}
$$

в котором $\delta>0$ - произвольное число, а постоянная $c_{\delta}$ не зависит от $\varepsilon \in\left(0, \varepsilon_{0}\right]$.

4. Случай $l_{0}=0$. Если $l_{0}$ удовлетворяет (1.13), то изложенная процедура построения асимптотики нуждается в модификации: необходимые условия разрешимости задачи (1.28) для определения коэффициентов $U_{k}$ ряда (1.32), вообще говоря, не выполняются.

Изменим структуру основного приближения. Именно, прибавим к (3.7) слагаемoe

$$
\varepsilon^{-1} c_{0}
$$

где $c_{0}$ - постоянная, подлежашая определению. Дополнительная невязка, порожденная (3.27) в (1.6), компенсируется еше одним слагаемьм типа пограничного слоя

$$
-\varepsilon^{-1} c_{0} \chi_{\Omega}(x) X\left(\varepsilon^{-1} x\right) .
$$

В (3.28) $X$ - введенное в п. $5 \S 2$ решение задачи $(1.29),(1.30)$ с правыми частями $\mathbf{F}=0, \mathbf{G}=1$, а $\chi_{\Omega}-$ та же срезка, что и в (3.6). K невязке, образованной функцией (3.28), применим процедуру перераспределения. Ту ее часть, которая составляет $o\left(\rho^{-2}\right)$, компенсируем членами $w_{0}, w_{1}, \ldots$ пограничного слоя, а остальные слагаемые отправим в уравнения $(1.22)$ и (1.28) для коэффициентов $v_{k}$ и $U_{k}$ ряда (1.32). Вспоминая структуру представления $X$ на бесконечности (см. формулы (2.23) и лемму 2.12), находим представление для невязки, образованной пограничньп слоем (3.28) и помещенной в уравнение (1.22):

$$
\varepsilon^{-1} H_{0}+\left\langle H_{1}\right\rangle_{\perp}+\cdots
$$

Здесь многоточием обозначены несущественные на данном этапе слагаемые,

$$
\begin{aligned}
H_{0}(x, y)= & -c_{0} C_{\omega}\left\{\left(L_{1}-L_{1}^{(0)}\right)\left(x, y, \partial_{x}, \partial_{y}\right)\left(\chi_{\Omega}(x) \Psi(x)\right)\right. \\
& \left.+\chi_{\Omega}(x)\left(L_{0}-L_{0}^{(0)}\right)\left(x, y, \partial_{y}\right)\left(\sum_{k=1}^{3} Y_{k}(0, y) \frac{\partial \Psi}{\partial x_{k}}(x)\right)\right\}, \\
H_{1}(x, y)= & c_{0} C_{\omega}\left\{\left[L_{1}, \chi_{\Omega}\right]\left(x, y, \partial_{y}\right) \sum_{k=1}^{3} Y_{k}(0, y) \frac{\partial \Psi}{\partial x_{k}}(x)\right. \\
& +\chi_{\Omega}(x)\left(L_{1}-L_{1}^{(0)}\right)\left(x, y, \partial_{x}, \partial_{y}\right)\left(\sum_{k=1}^{3} Y_{k}(0, y) \frac{\partial \Psi}{\partial x_{k}}(x)\right) \\
& \left.+\left[L_{2}, \chi_{\Omega}\right]\left(x, y, \partial_{x}\right) \Psi(x)+\chi_{\Omega}(x)\left(L_{2}-L_{2}^{(0)}\right)\left(x, y, \partial_{x}\right) \Psi(x)\right\} .
\end{aligned}
$$


Уравнение (1.22) с правой частью $H_{0}$ имеет решение $c_{0} V$, где

$$
V(x, y)=C_{\omega} \chi_{\Omega}(x) \sum_{k=1}^{3}\left(-Y_{k}(x, y)+Y_{k}(0, y)\right) \frac{\partial \Psi}{\partial x_{k}}(x) .
$$

Применяя процедуру перераспределения к невязке, образованной $c_{0} V$, получаем в правой части уравнения (1.22) для $v_{0}$ дополнительный член $\left\langle H_{2}\right\rangle_{\perp}$;

$$
H_{2}(x, y)=-c_{0} L_{1}\left(x, y, \partial_{x}, \partial_{y}\right) V(x, y) \text {. }
$$

В уравнение (1.28) для $U_{0}$ попадает сумма $\left\langle H_{1}\right\rangle+\left\langle H_{2}\right\rangle$. Согласно (3.29), (3.31) и $(1.21)$

$$
\begin{aligned}
\left\langle H_{1}\right\rangle+\left\langle H_{2}\right\rangle & =c_{0} C_{\omega}\left\{\mathbf{L}\left(\chi_{\Omega} \Psi\right)-\chi_{\Omega} \mathbf{L}^{0}(\Psi)\right\} \\
& =c_{0} C_{\omega}\left\{\chi_{\Omega}\left(\mathbf{L}-\mathbf{L}^{0}\right) \Psi+\left[\mathbf{L}, \chi_{\Omega}\right] \Psi\right\} .
\end{aligned}
$$

В самом деле, средняя часть (3.32) оказьвается обычной функцией (не распределением), и потому благодаря равенству $\int_{\Omega} \mathbf{L}\left(\chi_{\Omega} \Psi\right) d x=0$ (в смысле теории распределений) интеграл по $\Omega$ от любой из фигурных скобок в (3.32) равен -1 .

Таким образом, правая часть (3.5) наряду с функцией $\left\langle f^{(0)}\right\rangle$ содержит слагаемое (3.32), а условие разрешимости уравнения (3.5) выполняется в том случае, если

$$
c_{0}=C_{\omega}^{-1} \int_{\Omega} f^{(0)}(x) d x
$$

Итак, ряд (1.32) начинается со слагаемого, содержашего $\varepsilon^{-1}$, т.е. $\mu=-1$ в (1.32). Выясним погрешность в приближении решения $u(\varepsilon, x)$ только этим слагаемым

$$
\mathscr{U}_{-1}(\varepsilon, x)=\varepsilon^{-1} c_{0}\left\{1-\chi_{\Omega}(x) X\left(\varepsilon^{-1} x\right)+\varepsilon V\left(x, \varepsilon^{-1} x\right)\right\} .
$$

Как и в п. 2, для оценки $u-\mathscr{U}_{-1}$ введем величину

$$
\mathscr{V}_{-1}\left(x, \varepsilon^{-1} x\right)=\varepsilon^{-1} c_{0}\left\{1-\chi_{\Omega} X\left(\varepsilon^{-1} x\right)+\varepsilon\left(1-\chi_{\omega}\left(\varepsilon^{-1} x\right)\right) V\left(x, \varepsilon^{-1} x\right)\right\} .
$$

Разность $\mathscr{R}_{-1}=u-\mathscr{V}_{-1}$ удовлетворяет задаче

$$
\mathscr{L} \mathscr{R}_{-1}=\mathscr{F}_{-1} \quad \text { в } \Omega(\varepsilon), \quad \mathscr{R}_{-1}=0 \text { на } \partial \omega_{\varepsilon} .
$$

Оценка правой части $\mathscr{F}_{-1}$ производится по упрощенной схеме из п. 2. Отличия в появляюшихся далее весовых нормах вызваны новой априорной оценкой (1.14) при условии (1.13). 
Tеорема 3.5. В случае $l_{0}=0$ решение задачи (1.2), (1.5), (1.6) и его асимптотическое приближение (3.34) связаны неравенством

$$
\begin{aligned}
\varepsilon^{1 / 2}|| r^{-3 / 2}\left|\ln \frac{r}{a}\right|^{-1}\left(u-\mathscr{U}_{-1}\right) ; L_{2}(\Omega(\varepsilon)) \| & +\left\|\partial_{x}\left(u-\mathscr{U}_{-1}\right) ; L_{2}(\Omega(\varepsilon))\right\| \\
& \leqslant c\left\|u-\mathscr{U}_{-1} ; H^{1}(\Omega(\varepsilon))\right\| \leqslant c_{\delta} \varepsilon^{-1 / 2-\delta}
\end{aligned}
$$

в котором $\delta>0$ - произвольное число, а постоянная $c_{\delta}$ не зависит от $\varepsilon \in\left(0, \varepsilon_{0}\right]$.

Обрашаем внимание на то, что

$$
\left|\nabla_{x} \mathscr{U}_{-1}(\varepsilon, x)\right|=O\left(\varepsilon+\varepsilon^{-1}\left(\frac{r}{\varepsilon}\right)^{-2}\right),
$$

т.е. асимптотическое решение (3.34) не дает информации о поведении градиента решения: в качестве составляющей гладкого типа в (3.34) выступает постоянная (3.27). Для изучения асимптотики $\nabla_{x} \mathscr{U}_{-1}$ нужно, по крайней мере, учесть члены ряда (1.32) с номером $k=1$.

ЗАмечАниЕ 3.6. Аналогичные модификации нужны и в процедуре построения младших членов асимптотики решения задачи $(1.2),(1.5),(1.6)$ : условие разрешимости задачи (1.28) для нахождения $U_{k}$ обеспечивается дополнительными слагаемьми

$$
\varepsilon^{k-1} c_{k}\left\{1-\chi_{\Omega}(x) X\left(\varepsilon^{-1} x\right)+\varepsilon V\left(x, \varepsilon^{-1} x\right)\right\},
$$

относящимися к членам ряда (1.32) с номерами $k-1$.

\section{§4. Построение асимптотического разложения решения двумерной задачи Дирихле}

В силу следствия $2.11,2)$ решение плоской внешней задачи Дирихле $(1.29)$, (1.30), описывающей явление пограничного слоя, затухает на бесконечности только при выполнении дополнительного условия. Это обстоятельство вынуждает модифицировать процедуру построения асимптотики (тот же самый эффект наблюдается и при отсутствии быстрой осцилляции коэффициентов; ср. с [11], [21; §1.2], $[22 ; \S 2.4])$. Все необходимые изменения мы проследим на примере главных асимптотических членов в случаях $l_{0}>0$ и $l_{0}=0$. Нахождение младших членов и обоснование разложений не встречают новых затруднений.

1. Случай $l_{0}>0$. Как и в п. $1 \S 3$, основной член асимптотики решения задачи $(1.2),(1.5),(1.6)$ при $n=2$ ищем в виде (3.3). Слагаемое $U_{0}(x)$, являюшееся решением задачи (3.5), (1.5), ввиду следствия 2.3 в случае $n=2$ определяется по формуле

$$
U_{0}(x)=U_{0}^{0}(x)+A G(x),
$$

где $A$ - произвольная постоянная, а $G$ - функция Грина с особенностью в точке $O$,

$$
G(x)=\Psi(x)+G^{0}(x)
$$

$G^{0} \in C^{\infty}(\bar{\Omega})$ - ее регулярная часть, а $\Psi$ - фундаментальное решение (2.15) оператора $\mathbf{L}$. 
Поправка $v_{0}=v_{0 U}$ находится из уравнения (1.22) с правой частью (3.4). Поскольку $U_{0}$ имеет логарифмическую особенность, в отличие от трехмерного случая слагаемое $v_{0 U}$ не является гладкой функцией: согласно предложению 2.1 для $v_{0 U}$ справедливо представление $(2.2)$ с $\mu=-2$. Старший член $v_{0 U}^{(-2)}$ этого представления удовлетворяет уравнению

$$
\begin{aligned}
& L_{0}\left(0, y, \partial_{y}\right)\left(r^{-2} v_{0 U}^{(-2)}(\varphi, y)\right) \\
& \quad=-A\left(L_{2}\left(0, y, \partial_{x}\right) \Psi(x)+L_{1}\left(0, y, \partial_{x}, \partial_{y}\right) \sum_{p=1}^{2} Y_{p}(0, y) \frac{\partial \Psi}{\partial x_{p}}(x)\right)
\end{aligned}
$$

где $L_{0}\left(0, y, \partial_{y}\right)=M^{(0)}\left(y, \partial_{y}\right)$ - первый член ряда Тейлора (3.7). В (4.3) дифференцирование $\partial_{y}$ не затрагивает $x$ и $r, \varphi$, т.е. множитель $r^{-2}$ в (4.3) можно вынести за оператор $L_{0}$.

Вычисляя при учете (4.2) и (2.15) главную часть невязки функции (3.3) в краевом условии (1.6), заключаем, что поправка $w_{0}$ - решение задачи $(1.29),(1.30)$ с правыми частями

$$
\begin{aligned}
\mathbf{F}_{0}(y)= & \left(M^{0,2}(y, D)+M^{0,1}(y, D, \mathscr{D})\right)\left(\rho^{-2} v_{0}^{(-2)}(\varphi, y)\right) \\
& -A M^{0,2}(y, D)\left(\sum_{p=1}^{2} Y_{p}(0, y) \frac{\partial \Psi}{\partial y_{p}}(y)\right), \quad y \in \mathbb{R}^{2} \backslash \bar{\omega} \\
\mathbf{G}_{0}(y)= & -U_{0}^{0}(0)-A\left\{-\sigma \log (\varepsilon \rho)+\psi(\varphi)+G^{0}(0)+\sum_{p=1}^{2} Y_{p}(0, y) \frac{\partial \Psi}{\partial y_{p}}(y)\right\} \\
& -\rho^{-2} v_{0}^{(-2)}(\varphi, y), \quad y \in \partial \omega .
\end{aligned}
$$

ЗАмечАНИЕ 4.1. Напомним, что в трехмерном случае $\mathbf{F}_{0}=0$. Благодаря тому, что $\Psi(y)=\varepsilon \Psi(x)$ в $\mathbb{R}^{3}$, в $\S 3$ слагаемое вида (4.4) появлялось в правой части уравнения (1.22) для $v_{1}$.

Решение $w_{0}$ обязано затухать на бесконечности. Между тем, двумерная внешняя задача разрешима в классе функций, исчезающих на бесконечности, при условии

$$
\mathfrak{C}_{D}\left(\mathbf{F}_{0}, \mathbf{G}_{0}\right)=0
$$

(следствие $2.11,2)$ ). Оказывается, что для выполнения (4.6) можно использовать произвол в выборе решения (4.1) - коэффициент $A$. Именно с целью соблюсти условие (4.6) и был в (4.1) расширен класс допустимых решений: к ограниченному решению $U_{0}^{0} \in C^{\infty}(\bar{\Omega})$ задачи $(3.5),(1.5)$ добавлена функция Грина $G$. Множитель $A$, конечно же, вычисляется при помоши интегрального представления (2.19) функционала $\mathfrak{C}_{D}$. Однако сушествует более простой путь: ограниченное решение $w_{0}$ задачи $(1.29),(1.30)$ с правьми частями $(4.4),(4.5)$ указьвается явно:

$$
w_{0}(y)=-U_{0}^{0}(0)+A\left(\sigma \log \varepsilon-G^{0}(0)\right)+A(\zeta(y)-\mathscr{X}(y))-\rho^{-2} v_{0 U}^{(-2)}(\varphi, y) ;
$$

здесь $\zeta$ - построенное в п. $4 \S 2$ специальное решение (2.17), $\mathscr{X}$ - сумма (2.16). В согласии с асимптотическими формулами $(2.18),(2.11),(2.10)$ получаем

$$
(\zeta-\mathscr{X})(y)=C_{\omega}+\rho^{-1} Z(\varphi, \log \rho)+\sum_{p=1}^{2} Y_{p}(0, y) \frac{\partial}{\partial y_{p}}\left(\rho^{-1} Z(\varphi, \log \rho)\right)+\cdots
$$


где $C_{\omega}$ - интегральная характеристика, обсуждавшаяся перед предложением 2.10. Следовательно, для решения (4.7) типа пограничного слоя справедливо представление

$$
w_{0}(y)=-U_{0}^{0}(0)+A\left(\sigma \log \varepsilon-G^{0}(0)+C_{\omega}\right)+\cdots,
$$

в котором многоточием обозначен исчезающий при $y \rightarrow \infty$ остаток. Таким образом, функция $w_{0}$ обладает необходимьм свойством затухания на бесконечности, если

$$
A=A(\log \varepsilon)=-U_{0}^{0}(0) \mu(\log \varepsilon),
$$

где $\mu(\log \varepsilon)=\left\{\sigma|\log \varepsilon|+G^{0}(0)-C_{\omega}\right\}^{-1}$. Подчеркнем, что $\mu(\log \varepsilon)=O\left(|\log \varepsilon|^{-1}\right)$, т.е. при достаточно малом $\varepsilon>0$ выражение из фигурных скобок положительно.

Итак, предъявлены слагаемые $U_{0}$ и $w_{0}$ в конструкции главного члена асимптотики решения $u$ задачи $(1.2),(1.5),(1.6)$ при $n=2$; осталось найти функцию $v_{0}$. Как и в трехмерном случае (cp. с п. $1 \S 3)$, пограничный слой $w_{0}$ порождает дополнительную невязку

$$
\begin{aligned}
\sum_{j, k=1}^{2}\left\{A \frac{\partial l_{j k}}{\partial y_{j}}(x, y) r^{-1} Z\left(\varphi, \log \left(\varepsilon^{-1} r\right)\right) \frac{\partial \chi}{\partial x_{k}}(x)\right. \\
+\chi(x) \frac{\partial}{\partial y_{j}}\left(l_{j k}(x, y)-l_{j k}(0, y)\right)\left(A \frac{\partial}{\partial x_{k}} r^{-1} Z\left(\varphi, \log \left(\varepsilon^{-1} r\right)\right)\right. \\
\left.\left.\quad+A \sum_{p=1}^{2} \frac{\partial Y_{p}}{\partial y_{k}}(0, y) \frac{\partial}{\partial x_{p}} r^{-1} Z\left(\varphi, \log \left(\varepsilon^{-1} r\right)\right)-\frac{\partial v_{0 U}^{(-2)}}{\partial y_{k}}(\varphi, y)\right)\right\}
\end{aligned}
$$

Она компенсируется решением $v_{\text {ow }}$ уравнения (1.22). Таким образом, быстроосциллирующий корректор $v_{0}$ состоит из двух слагаемых $v_{0 U}$ и $v_{0 w}$, являющихся решениями уравнения (1.22) с правыми частями (3.4) и (4.10) соответственно.

Построение следуюших членов асимптотического ряда (1.32) проводится по прежней схеме, включающей процедуру перераспределения невязок и подобное (4.1) представление $U_{k}=U_{k}^{0}+A_{k} G$ решения гладкого типа. Единственное отличие от случая $k=0$ состоит в усложнении зависимостей коэффициентов от $\log \varepsilon$; в частности, оказывается, что $A_{k}(\log \varepsilon)=\mu(\log \varepsilon)^{-k} A_{k}^{0}(\log \varepsilon)$, где $A_{k}^{0}(l)$ - полиHOM.

2. Случай $l_{0}=0$. Задача $(3.5),(1.5)$, вообше говоря, неразрешима (см. предложение 1.5). Поступим так же, как и в п. 1, - расширим класс допустимых решений за счет растущих логарифмически при $x \rightarrow 0$. Иными словами, будем искать функцию $U_{0}$ в виде

$$
U_{0}(x)=U_{0}^{0}(x)+c_{0}+c_{1} \chi_{\Omega}(x) \Psi(x) .
$$

Постоянная $c_{0}$ в настоящий момент произвольна, а множитель $c_{1}$ фиксируется так:

$$
c_{1}=-\int_{\Omega}\left\langle f_{0}\right\rangle(x) d x
$$

Наконец, $U_{0}^{0} \in C^{\infty}(\bar{\Omega})$ - решение уравнения

$$
\mathbf{L}_{0} U_{0}^{0}=\left\langle f_{0}\right\rangle-c_{1}\left[\mathbf{L}_{0}, \chi_{\Omega}\right] \Psi \quad \text { в } \quad \Omega
$$


с условиями периодичности (1.5). Благодаря (4.12) условие разрешимости задачи $(4.13),(1.5)$ удовлетворено (см. предложение $1.2,1))$. Так как решение $U_{0}^{0}$ определено с точностью до аддитивной постоянной, его можно нормировать равенством $U_{0}^{0}(0)=0$.

Отыскивая главную часть невязки суммы (4.11) в краевом условии (1.6), выгисляем правые части задачи $(1.29),(1.30)$ для поправки типа пограничного слоя $w_{0}$; они имеют вид $(4.4),(4.5)$, где $A$ и $U_{0}^{0}$ заменены соответственно на $c_{1}$ и $c_{0}$, а величина $G^{0}(0)$ считается равной нулю (к такому выводу можно прийти, сравнив $(4.1)$ и (4.11)). Таким образом, аналогично (4.7)

$$
w_{0}(y)=-c_{0}+c_{1} \sigma \log \varepsilon+c_{1}(\zeta(y)-\mathscr{X}(y))-\rho^{-2} v_{0 U}^{(-2)}(\varphi, y) .
$$

Функция (4.14) ограничена и затухает при $y \rightarrow \infty$ в случае

$$
c_{0}=c_{1} \sigma \log \varepsilon-c_{1} C_{\omega} .
$$

Это равенство ликвидирует произвол в (4.11). Последняя составляющая главного члена - быстроосциллирующий корректор $v_{0}$ - находится так же, как и при $l_{0}>0$ (см. п. 1).

Ввиду “накопления логарифмов" из-за переходов от медленных переменных к быстрым и наоборот младшие члены ряда (1.32) становятся полиномами от $\log \varepsilon$ высших степеней.

\section{§5. Обобщения}

1. Задача Неймана. В большинстве случаев замена условий Дирихле (1.6) условиями Неймана (1.7) не приводит к каким-либо осложнениям, но требует введения новой интегральной характеристики, описанной в п. $6 \S 2$. При $l_{0}>0$ итерационные процессы построения полной асимптотики решения трехмерной задачи (1.2), (1.5), (1.7) полностью совпадают с процессами из §3, а для двумерной задачи - даже упрощаются (так как постоянная удовлетворяет однородному условию (1.31), то рациональной зависимости от $\log \varepsilon$ в (4.9) не возникает). Если же $l_{0}=0$, то у исходной и осредненной задач имеются условия разрешимости, выполнение которых приходится отслеживать на каждом шаге алгорифма. Исследование влияния сингулярных возмущений границы на условия разрешимости задач с гладкими (без осцилляции) коэффициентами проводилось в [15] (см. также $[22],[23])$.

2. Оценки старших производных. Предложение 1.3 позволяет оценить соболевские нормы разности истинного и асимптотического решений, однако из-за специфики рассматриваемой задачи пространства Соболева снабжаются нормами, зависящими от $\varepsilon$ (ср. с [41], [42] и др.). Так, например, улучшенная оценка (3.18) выглядит следующим образом:

$$
\left\|r^{-1}\left(u_{0}-\mathscr{U}_{0}\right) ; L_{2}(\Omega(\varepsilon))\right\|+\sum_{1 \leqslant|\alpha| \leqslant l+2} \varepsilon^{|\alpha|-1}\left\|\partial_{x}^{\alpha}\left(u_{0}-\mathscr{U}_{0}\right) ; L_{2}(\Omega(\varepsilon))\right\| \leqslant c_{l, \delta} \varepsilon^{1-\delta} .
$$

На первый взгляд кажется, что появление степеней $\varepsilon$ с положительными показателями при старших производных разности $u_{0}-\mathscr{U}_{0}$ делает оценку (5.1) бесполезной. Это впечатление ошибочно, поскольку из-за быстроосциллирующих корректоров 
и пограничных слоев производная $\partial_{x}^{\alpha} \mathscr{U}_{0}(\varepsilon, x)$ сама оказывается большей; именно, согласно (3.16) при $|\alpha|>0$

$$
\left\|\partial_{x}^{\alpha} \mathscr{U}_{0} ; H^{1}(\Omega(\varepsilon))\right\| \sim c\left(\int_{\Omega(\varepsilon)}\left(\varepsilon^{1-|\alpha|}+\varepsilon^{-|\alpha|}\left(\frac{r}{\varepsilon}\right)^{|\alpha|}\right)^{2} d x\right)^{1 / 2} \sim C \varepsilon^{1-|\alpha|} .
$$

Теперь видно, что неравенство (5.1) обосновывает малость относительной погрешности приближения $\partial_{x}^{\alpha} u_{0}(\varepsilon, x)$ производной $\partial_{x}^{\alpha} \mathscr{U}_{0}(\varepsilon, x)$ и что распределение степеней $\varepsilon$ справа в (5.1) следует признать правильным. Те же приемы, что и в доказательстве предложения 1.3, вместе с общими результатами [43] приводят к априорным оценкам решений в классах Гёльдера. В частности, в дополнение к теореме 3.3 и следствиям из нее можно получить такую оценку:

$$
\left|\nabla_{x} u(\varepsilon, x)-\nabla_{x}\left\{U_{0}(x)+\varepsilon \sum_{k=1}^{3} Y_{k}\left(x, \frac{x}{\varepsilon}\right) \frac{\partial U_{0}}{\partial x_{k}}(x)-U_{0}(0) X\left(\frac{x}{\varepsilon}\right)\right\}\right| \leqslant c_{\delta} \varepsilon^{1-\delta} .
$$

3. Системы уравнений. Асимптотические конструкции и процессы, подготовленные в данной статье, пригодны и для общих самосопряженных эллиптических краевых задач. В части обоснования ограничительным является тот факт, что в [30], [31] изучены только системы второго порядка. Вместе с тем, перенесение результатов на задачи теории упругости не встречает затруднений при сохранении условий периодичности типа (1.5). Все члены рядов становятся вектор-функциями, и потому для двумерной задачи в п. $1 \S 4$ неизвестный вектор $A(\log \varepsilon)$ находится при решении системы линейных алгебраических уравнений, т.е.

$$
A(\log \varepsilon)=P_{1}(\log \varepsilon) P_{2}(\log \varepsilon)^{-1}, \quad \operatorname{deg} P_{i}=i, \quad P_{1}=\left(P_{11}, P_{12}\right) .
$$

4. Другие типы локальных возмущений. Предложенный подход годится и тогда, когда в $\Omega$ появляется малое инородное включение, т.е. уравнение (1.2) выполняется всюду в $\Omega$, но его коэффициенты имеют вид

$$
\begin{aligned}
& l_{i j}(\varepsilon, x)=l_{i j}^{0}\left(x, \varepsilon^{-1} x\right), \quad x \in \Omega \backslash \omega_{\varepsilon} ; \\
& l_{i j}(\varepsilon, x)=l_{i j}^{1}\left(x, \varepsilon^{-1} x\right), \quad x \in \omega_{\varepsilon} .
\end{aligned}
$$

Подчеркнем, что такая задача сохраняет все свойства задачи Неймана. Наконец, при помощи малозначительных модификаций итерационных процессов можно рассмотреть случай $l_{i j}^{1}(\varepsilon, y)=\varepsilon^{m} l_{i j}^{2}(\varepsilon, y)$ в $(5.4)$; здесь $m$ - целое (положительное или отрицательное) число.

5. Несколько отверстий. Обсудим задачу $(1.2),(1.5),(1.6)$ в области $\Omega(\varepsilon)$, получающейся удалением из параллелепипеда $\Omega$ нескольких малых отверстий $\omega_{\varepsilon}^{1}, \ldots, \omega_{\varepsilon}^{N}$ с диаметрами $O(\varepsilon)$ и центрами $P^{1}, \ldots, P^{N}$, удаленньпи один от другого на расстояние $O(1)$ (если расстояние между двумя отверстиями есть $O(\varepsilon)$, то их следует рассматривать как единое целое - связность множества $\bar{\omega}$ в статье не использовалась). В случае $n=3, l>0$ итерационные процессы сохраняются, и только последнее слагаемое в (1.32) приходится заменить суммой

$$
\sum_{m=1}^{N} \chi_{m}(x) w_{k}^{m}\left(\varepsilon^{-1}\left(x-P^{m}\right), \log \varepsilon\right)
$$


(срезки $\chi_{m}$ нужно выбрать так, чтобы supp $\chi_{m}$ не содержал точек $P^{1}, \ldots, P^{m-1}$, $\left.P^{m+1}, \ldots, P^{N}\right)$. В двумерных задачах размножение пограничных слоев по формуле (5.5) сопровождается усложнением зависимостей коэффициентов от $\log \varepsilon$. Так, в случае $l>0$ они оказываются дробями вида $P_{N-1}(\log \varepsilon) P_{N}(\log \varepsilon)^{-1}$.

6. Краевые условия на гранях параллелепипеда. Пусть на двух противоположных гранях $\Gamma_{i}^{ \pm}$параллелепипеда $\Omega$ заданы условия Дирихле или Неймана, а на остальных - условия периодичности (1.5). В соответствующей задаче для уравнения (1.2) можно построить полное асимптотическое разложение решения, так как явление пограничного слоя около прямолинейного участка границы изучено полностью (см. [4]-[7], [44]-[46] и др.). Именно, предельные задачи, разобранные в $\S 2$, дополняются еще одной задачей, которая содержит уравнение с оператором $\mathscr{L}\left(x^{0}, y, \partial_{y}\right)$ в полубесконечной призме $\mathbb{P}=[0,1]^{n-1} \times \mathbb{R}_{+}$, а также краевые условия Дирихле или Неймана на основании и условия периодичности на боковых гранях призмы. Требование экспоненциального затухания на бесконечности решений этой задачи порождает краевые условия на $\Gamma_{i}^{ \pm}$, которыми снабжается осредненная задача (1.28) в $\Omega$, а сами дефектные функционалы, обеспечивающие затухание, исследовались в работах [4], [47]-[51] и др. (см. также гл. 3, 5 в книгах [28] и [29]).

Простота асимптотических конструкций, упомянутых в предыдущем абзаце, объясняется возможностью продолжить по периодичности данные задачи с $\Omega$ на слой, ограниченньй гиперплоскостями $\left\{x \in \mathbb{R}^{n}: x_{i}= \pm a_{i}\right\}$. По-иному дела обстоят в случае постановки краевых условий на гранях, примькающих одна к другой. Из-за возникновения настоящей (не устраняемой периодическим продолжением) реберной особенности границы появляются дополнительные степенные пограничные слои, которые изучены только в двумерной ситуации (см. [37], [52]). Опять-таки, наиболее серьезное препятствие - исследование поведения около бесконечности решений задач с периодическими коэффициентами в угловых областях. Как и в п. 2, алгорифмы [37], [52] без особых усилий могут быть приспособлены к задаче Дирихле для системы уравнений теории упругости в прямоугольнике, но обобщения на системы сложной структуры, иные краевые условия или многомерные области пока недоступны.

\section{Список литературы}

1. Ладыженская О. А. Краевые задачи математической физики. М.: Наука, 1973.

2. Лионс Ж.-Л., Мадженес Э. Неоднородные граничные задачи и их приложения. М.: Мир, 1971.

3. Bensoussan A., Lions J.-L., Papanicolaou G. Asymptotic analysis for periodic structures. Amsterdam: North-Holland, 1978.

4. Lions J.-L. Some methods in the mathematical analysis of systems and their control. Beijing, China: Science Press, 1981.

5. Бахвалов Н. С., Панасенко Г. А. Осреднение процессов в периодических средах. М.: Наука, 1984.

6. Санчес-Паленсия Э. Неоднородные среды и теория колебаний. М.: Мир, 1984.

7. Олейник О.А., Иосифьлн Г. А., Шамаев А. С. Математические задачи теории сильо неоднородных сред. М.: Изд-во МГУ, 1990.

8. Жиков В. В., Козлов С.М., Олейник О.А. Усреднение дифференциальных операторов. М.: Наука, 1993.

9. Берс Л., Джсон Ф., Шехтер М. Уравнения с частными производньми. М.: Мир, 1968. 
10. Ильин A. М. Краевая задача для эллиптических уравнений второго порядка в области с щелью. 1. Двумерньй случай // Матем. сб. 1976. Т. 99. № 4. С. 514-537.

11. Ильин A. М. Краевая задача для эллиптических уравнений второго порядка в области с щелью. 2. Область с малым отверстием // Матем. сб. 1977. Т. 103. № 2. С. 265-284.

12. Ильин А. М. Исследование асимптотики решения эллиптической краевой задачи в области с мальм отверстием // Труды сем. им. И.Г. Петровского. №6. М.: Изд-во МГУ, 1981. С. $57-82$.

13. Ильин A. M. Согласование асимптотических разложений решений краевых задач. М.: Наука, 1989.

14. Мазья В.Г., Назаров С.А., Пламеневский Б. А. Об асимптотике решений эллиптических краевых задач при вариации области вблизи конических точек // Докл. АН CCCP. 1979. T. 249. №1. С. 94-96.

15. Мазья В. Г., Назаров С.А., Пламеневский Б.А. Об асимптотике решений эллиптических краевых задач при нерегулярном возмущении области // Проблемы матем. анализа. № 8. Л.: Изд-во ЛГУ, 1981. С. 72-153.

16. Мазья В. Г., Назаров С.А., Пламеневский Б. А. Асимптотические разложения собственных чисел краевых задач для оператора Лапласа в областях с мальми отверстиями // Изв. АН СССР. Сер. матем. 1984. Т. 48. № 2. С. 347-371.

17. Мазья В. Г., Назаров С. А., Пламеневский Б. А. Об особенностях решений задачи Дирихле во внешности тонкого конуса // Матем. сб. 1983. Т. 122. № 4. С. 435-456.

18. Мазья В. Г., Назаров С. А. Асимптотика интегралов энергии при малых возмущениях границы вблизи угловых и конических точек // Труды ММО. 1987. Т. 50. С. 79-129.

19. Мазья В.Г., Назаров С.А. Об особенностях решений задачи Неймана в конической точке // Сиб. матем. журн. 1989. Т. 30. №3. С. 52-63.

20. Козлов В.А., Мазья В.Г. О спектре операторного пучка, порожденного задачей Дирихле в конусе // Матем. сб. 1991. Т. 182. № 5. С. 638-660.

21. Nazarov $S$. A. Interaction of concentrated masses in a harmonically oscillating spatial body with Neumann boundary conditions // Modelisation Math. Anal. Numer. 1993. V. 27. № 6 . P. 777-799.

22. Мазья В.Г., Назаров С. А., Пламеневский Б. А. Асимптотика решений эллиптических краевых задач при сингулярных возмущениях области. Тбилиси: Изд-во ТГУ, 1980.

23. Mazja W. G., Nasarow S.A., Plamenewski B. A. Asymptotische Theorie elliptischer Randwertaufgaben in singulär gestörten Gebieten. V. 1. Berlin: Akademie-Verlag, 1991.

24. Кондратьев В. А. Краевые задачи для эллиптических уравнений в областях с коническими или угловыми точками // Труды ММО. 1967. Т. 16. С. 209-298.

25. Pazy A. Asymptotic expansions of solutions of ordinary differential equations in Hilbert space // Arch. Rational Mech. Anal. 1967. V. 24. P. 193-218.

26. Мазья В. Г., Пламеневский Б. А. О коэффициентах в асимптотике решений эллиптических краевых задач в области с коническими точками // Math. Nachr. 1977. V. 76. P. 29-60.

27. Кондратьев B. A., Олейник O.A. Краевые задачи для системы теории упругости в неограниченных областях. Неравенство Корна // УМН. 1988. Т. 43. № 5. С. 55-98.

28. Назаров С. А., Пламеневский Б. А. Эллиптические задачи в кусочногладких областях. М.: Наука, 1991.

29. Nazarov S.A., Plamenevsky B.A. Elliptic problems in domains with piecewise smooth boundaries. Berlin: Walter de Gruyter, 1994.

30. Назаров С. А. Задача Дирихле для эллиптической системы с периодическими коэффициентами в угловой области // Вестн. ЛГУ. 1990. № 1. С. 32-35.

31. Nazarov S. A. Asymptotics at infinity of the solution to the Dirichlet problem for a system of equations with periodic coefficients in an angular domain // Russian J. Math. Phys. 1995. V. 3. № 3. P. 297-325.

32. Бахвалов Н.С. Осреднение дифференциальных уравнений с быстроосциллирующими коэффициентами // Докл. АН СССР. 1975. Т. 221. №3. С. 516-519.

33. Сандраков Г. В. Принципы осреднения уравнений с быстроосциллирующими коэффициентами // Матем. сб. 1989. Т. 180. № 12. С. 1634-1679. 
34. Назаров С. А. Общая схема осреднения самосопряженных эллиптических систем в многомерных областях, в том числе тонких // Алгебра и анализ. 1995. Т. 7. № 5. С. 1-92.

35. Слуцкий A.C. Степенной пограничный слой в задаче осреднения вырождающегося эллиптического уравнения // Деп. в ВИНИТИ №3358-В86, 1986.

36. Мовчан А.Б., Назаров С.А. Трещины в композитных материалах. 1 // Механика композитных материалов. 1990. №5. С. 842-851; 2 // Механика композитных материалов. 1990. №6. С. 1038-1046.

37. Назаров C. A. Асимптотика решений задачи Дирихле для уравнений с быстро осциллирующими коэффицциентами в прямоугольнике // Матем. сб. 1991. Т. 182. № 5. С. 692-722.

38. Кондратьев B. A., Олейник O. А. Об асимптотике на бесконечности решений с конечным интегралом Дирихле для эллиптических уравнений второго порядка // Труды сем. им. И.Г. Петровского. № 12. М.: Изд-во МГУ, 1987. С. 149-163.

39. Полиа Г., Сеге Г. Изопериметрические неравенства в математической физике. М.: Физматгиз, 1962.

40. Ланскоф Н. С. Основы современной теории потенциала. М.: Наука, 1966.

41. Агранович M. С., Вишик М.И. Эллиптические задачи с параметром и параболические задачи общего вида // УМН. 1964. Т. 19. № 3. С. 53-160.

42. Назаров С. А. Метод Вишика-Люстерника для эллиптических краевых задач в областях с коническими точками. 1. Задача в конусе // Сиб. матем. журн. 1981. Т. 22. № 4. C. $142-163$.

43. Агмон $C$. , Дуглис А., Ниренберг Л. Оценки решений эллиптических уравнений вблизи границы. М.: ИЛ, 1962.

44. Панасенко Г. П. Асимптотики высших порядков решений задач о контакте периодических структур // Матем. сб. 1979. Т. 110. № 4. С. 505-538.

45. Олейник О.А., Иосифьян Г. А., Панасенко Г. П. Асимптотическое разложение решений системы теории упругости в перфорированных областях // Матем. сб. 1983. Т. 120. № 1. C. $22-41$.

46. Oleinik O.A., Panasenko G.P., Yosifian G.A. On the asymptotic expansion for solution of a homogenization problem in elasticity // Appl. Anal. 1983. V. 15. № 1-4. P. 15-32.

47. Ландис Е. М., Панасенко Г. П. Теорема об асимптотике решений эллиптических уравнений с коэффициентами, периодическими по всем переменньп, кроме одной // Докл. АН CCCP. 1977. T. 235. №6. C. 1253-1255.

48. Назаров С. А. Эллиптические краевые задачи с периодическими коэффициентами в цилиндре // Изв. АН СССР. Сер. матем. 1981. Т. 45. №1. С. 101-112.

49. Назаров C.A. О коэффициентах в асимптотике решений эллиптических краевых задач с периодическими коэффициентами // Вестн. ЛГУ. 1985. № 15. С. 16-22.

50. Олейник O.A., Иосифьян $Г . A$. Об условиях затухания и предельном поведении решений системы уравнений теории упругости // Докл. АН СССР. 1981. Т. 258. №3. C. $550-553$.

51. Oleinik O.A., Yosifian G.A. On the asymptotic behavior at infinity of solutions in linear elasticity // Arch. Rational Mech. Anal. 1982. V. 78. № 1. P. 29-53.

52. Назаров С.A. Асимптотика решений задачи Дирихле в прямоугольнике для уравнения с быстроосциллирующими коэффициентами // УМН. 1985. Т. 40. № 5. С. 219-220.

Государственная морская академия им. адм. С. О. Макарова,

Поступила в редакцию Санкт-Петербург;

Институт проблем машиностроения РАН, 16.12 .1996 и 15.06 .1998

Санкт-Петербург 